\title{
SUFFICIENT OPTIMALITY CONDITIONS AND SEMI-SMOOTH NEWTON METHODS FOR OPTIMAL CONTROL OF STATIONARY VARIATIONAL INEQUALITIES
}

\author{
Karl Kunisch $^{1}$ AND DANiel Wachsmuth ${ }^{2}$
}

\begin{abstract}
In this paper sufficient second order optimality conditions for optimal control problems subject to stationary variational inequalities of obstacle type are derived. Since optimality conditions for such problems always involve measures as Lagrange multipliers, which impede the use of efficient Newton type methods, a family of regularized problems is introduced. Second order sufficient optimality conditions are derived for the regularized problems as well. It is further shown that these conditions are also sufficient for superlinear convergence of the semi-smooth Newton algorithm to be well-defined and superlinearly convergent when applied to the first order optimality system associated with the regularized problems.
\end{abstract}

Mathematics Subject Classification. 49K20, 47J20, 49M15.

Received July 14, 2010. Revised February 2, 2011.

Published online July 22, 2011.

\section{Introduction, Problem statement, REgularization}

We consider the optimal control problem:

$$
\left\{\begin{array}{l}
\min \quad J(y, u)=g(y)+j(u) \\
\text { over } u \in L^{2}(\Omega) \text { under the variational inequality constraint } \\
a(y, \phi-y) \geq(u, \phi-y), \quad y \in K, \text { for all } \phi \in K
\end{array}\right.
$$

where $a: H_{0}^{1}(\Omega) \times H_{0}^{1}(\Omega) \rightarrow \mathbb{R}$ is a bilinear form satisfying

$$
\nu_{1}|v|_{H_{0}^{1}}^{2} \leq a(v, v), \text { and } a(v, w) \leq \nu_{2}|v|_{H_{0}^{1}}|w|_{H_{0}^{1}}
$$

with $0<\nu_{1} \leq \nu_{2}$, and

$$
K=\left\{v \in H_{0}^{1}(\Omega): v \leq \psi\right\}
$$

\footnotetext{
Keywords and phrases. Variational inequalities, optimal control, sufficient optimality conditions, semi-smooth Newton method.

1 Institute for Mathematics and Scientific Computing, Heinrichstraße 36, 8010 Graz, Austria. karl.kunisch@uni-graz.at, http://www.kfunigraz.ac.at/imawww/kunisch

Partially supported by "Fonds zur Förderung der Wissenschaftlichen Forschung" under SFB 32, Mathematical Optimization and Applications in the Biomedical Sciences.

2 Johann Radon Institute for Computational and Applied Mathematics (RICAM), Austrian Academy of Sciences, Altenbergerstraße 69, 4040 Linz, Austria. daniel.wachsmuth@oeaw.ac.at, http://www.ricam.oeaw.ac.at/people/page/wachsmuth
} 
Throughout the paper it will be convenient to alternatively use the operator representation of the bilinear form, i.e.

$$
a\left(v_{1}, v_{2}\right)=\left\langle A v_{1}, v_{2}\right\rangle, \text { for } v_{1}, v_{2} \in H_{0}^{1}(\Omega),
$$

where $\langle\cdot, \cdot\rangle$ stands for the duality pairing between $H^{-1}(\Omega)$ and $H_{0}^{1}(\Omega)$. It is well known that under the conditions to be specified below the variational inequality in $(\mathrm{P})$ can equivalently be expressed as

$$
A y+\lambda=u, \quad y \leq \psi, \lambda \geq 0,(\lambda, y-\psi)_{L^{2}}=0,
$$

where $\lambda \in L^{2}$ and $\psi \in H_{0}^{1}(\Omega)$. In this way (P) is an optimization problem subject to a complementary condition constraint. Since $\lambda \in L^{2}(\Omega)$, the complementarity condition in (1.3) can equivalently be expressed as

$$
\lambda=\max (0, \lambda+c(y-\psi)),
$$

for any $c>0$.

If the bilinear form $a$ is symmetric, the variational inequality constraint is also equivalent to the optimization problem

$$
\min \frac{1}{2} a(y, y)-(y, u)_{L^{2}} \text { over } y \in K,
$$

so that $(\mathrm{P})$ can alternatively be considered as a bilevel optimization problem with the additional constraint $y \in K$. Here $(\cdot, \cdot)_{L^{2}}$ denotes the scalar product in $L^{2}(\Omega)$. In the sequel the index $L^{2}$ will frequently be omitted.

Let us describe the structure and contributions of this paper. Section 2 is devoted to optimality conditions for $(\mathrm{P})$. While the focus is put on sufficient optimality conditions, we shall also recapture first order necessary optimality conditions. In this respect the literature essentially offers two approaches. One is based on convex analysis techniques, see for instance [16], the other one on approximation methods, we refer to [1,12], and further references given there. The main concern here is to obtain a system of conditions as complete as possible, so that its solutions in turn provide the solution to $(\mathrm{P})$, see e.g. [3]. Problem (P) can be viewed as multi-level optimization problem. While such problems have received repeated attention in the finite-dimensional context, see for instance [19], this point of view is not common in the infinite dimensional setting. See, however, the recent contribution [9]. To the authors' knowledge, sufficient conditions for control of variational inequalities were not investigated yet. The only result in this context is due to [15]: there it is proven that $(\mathrm{P})$ is convex if $\psi \leq y_{d}$, which means that the desired state is "behind" the obstacle. Let us point out the related work on sufficient optimality conditions for control of state-constrained optimal control problems, in e.g. [5-7,17,18].

In fact, it is worthwhile to compare the optimal control problem considered here, to the following optimal control problem subject to state constraints:

$$
\left\{\begin{array}{l}
\min J(y, u)=g(y)+j(u) \\
\text { over } u \in L^{2}(\Omega), y \in K, \text { under the elliptic equation } \\
A y=u
\end{array}\right.
$$

If the bilinear form $a$ is symmetric (or $A$ is self-adjoint), both problems can be expressed as bilevel optimization problems. Problem (P) can be written as:

$$
\begin{aligned}
& \min J(y, u) \text { over all } u \in L^{2}(\Omega) \text { and } y \in H_{0}^{1}(\Omega) \text { with } \\
& y=\operatorname{argmin}_{y \in K} \frac{1}{2} a(y, y)-(y, u)_{L^{2}},
\end{aligned}
$$

whereas the state constrained problem can be written as

$$
\begin{aligned}
& \min J(y, u) \text { over all } u \in L^{2}(\Omega) \text { and } y \in K \text { with } \\
& y=\operatorname{argmin}_{y \in H_{0}^{1}(\Omega)} \frac{1}{2} a(y, y)-(y, u)_{L^{2}} .
\end{aligned}
$$


The essential difference between both problems is now evident: In the variational inequality control problem, the constraint $y \leq \psi$ is prescribed in the inner problem, whereas for the state constrained problem this constraint appears in the outer problem. This implies that for $(\mathrm{P})$ every control is feasible, whereas for the state constrained problem the inequality $y \leq \psi$ is in fact a restriction on the set of feasible controls. Consequently the first-order necessary optimality conditions differ as well. Problem (P) relies on two multipliers associated to the constraint $y \in K$, denoted by $\lambda$ and $\mu$ below, where one, $\lambda$, is non-negative. The state-constrained problem involves only one multiplier for $y \in K$, which is a non-negative measure.

In Section 3 we investigate properties of a regularization of $(\mathrm{P})$. This does justice to the fact that the Lagrange multiplier $\lambda$ associated to the inequality constrained $y \leq \psi$ as well as the multiplier, which will be called $\mu$ below and is associated to the complementary system in (1.3), are measures only, and such constitute quantities that are not amenable to numerical discretization and realisation. The regularized problems that will be utilized are given by

$$
\left\{\begin{array}{l}
\min J(y, u)=g(y)+j(u) \\
\text { over } u \in L^{2}(\Omega) \text { subject to } \\
A y+\max _{c}(\bar{\lambda}+c(y-\psi))=u,
\end{array}\right.
$$

where $\bar{\lambda} \geq 0, \bar{\lambda} \in L^{2}(\Omega)$ is given, and $\max _{c}$ is the $C^{1}$-approximation of $x \rightarrow \max (0, x)$ constructed by

$$
\max _{c}(0, x)= \begin{cases}x, & \text { for } x \geq \frac{1}{2 c} \\ \frac{c}{2}\left(x+\frac{1}{2 c}\right)^{2}, & \text { for }|x| \leq \frac{1}{2 c} \\ 0, & \text { for } x \leq-\frac{1}{2 c}\end{cases}
$$

For properly chosen $\bar{\lambda}$ the solutions $y_{c}$ to $\left(\mathrm{P}_{c}\right)$ are feasible, i.e. $y_{c} \leq \psi$, see Section 3.2 below. This was observed in [12] and will be further analysed and used in the present paper. If $g$ and $j$ are $C^{1}$-regular, then the first order optimality system for $\left(\mathrm{P}_{c}\right)$ is given by

$$
\left\{\begin{array}{l}
A y_{c}+\max _{c}\left(\bar{\lambda}+c\left(y_{c}-\psi\right)\right)=u_{c} \\
A^{*} p_{c}+c \operatorname{sgn}_{c}\left(\bar{\lambda}+c\left(y_{c}-\psi\right)\right) p_{c}+g^{\prime}\left(y_{c}\right)=0, \\
j^{\prime}\left(u_{c}\right)-p_{c}=0
\end{array}\right.
$$

where

$$
\operatorname{sgn}_{c}(x)= \begin{cases}1, & \text { for } x \geq \frac{1}{2 c} \\ c\left(x+\frac{1}{2 c}\right), & \text { for }|x| \leq \frac{1}{2 c} \\ 0, & \text { for } x \leq-\frac{1}{2 c}\end{cases}
$$

and the expressions $\lambda_{c}:=\max _{c}\left(\bar{\lambda}+c\left(y_{c}-\psi\right)\right)$ and $\mu_{c}:=c \operatorname{sgn}_{c}\left(\bar{\lambda}+c\left(y_{c}-\psi\right)\right) p_{c}$ in (1.7) tend to measurevalued Lagrange multipliers as $c \rightarrow \infty$. Section 3 also contains a discussion of second order sufficient optimality conditions for $\left(\mathrm{P}_{c}\right)$.

Solving (1.7) numerically by Newton-type methods is impeded by the lack of $C^{1}$ regularity of the $\operatorname{sgn}_{c}$ operator. In Section 4 it will be shown that semi-smooth Newton methods are applicable to (1.7) [14]. This requires the verification of Newton differentiability, which is quite standard by now, as well as well-posedness of the Newton-step and uniform boundedness of the inverse of the generalized derivatives, which is more delicate to verify. Here this will be achieved under a second-order sufficient optimality condition.

In a followup paper we address numerical issues related to solving $\left(\mathrm{P}_{c}\right)$ and in particular on the choice of the parameter $c$. This will make use of the properties of the path associated to $\left(\mathrm{P}_{c}\right)$, i.e. on the mapping $c \rightarrow\left(y_{c}, u_{c}, \lambda_{c}\right)$. 


\section{Standing assumptions}

Throughout the paper we rely on the following regularity assumptions.

(i) The domain $\Omega \subset \mathbb{R}^{n}, n \in\{2,3\}$ is bounded, and its boundary is of class $C^{1,1}$.

(ii) The operator $A$ is an elliptic differential operator defined by

$$
(A y)(x)=-\sum_{i, j=1}^{n} \frac{\partial}{\partial x_{i}}\left(a_{i j}(x) \frac{\partial}{\partial x_{j}} y(x)\right)+\sum_{j=1}^{n} a_{j}(x) \frac{\partial}{\partial x_{j}} y(x)+a_{0}(x) y(x)
$$

with functions $a_{i j} \in C^{0,1}(\bar{\Omega}), a_{j}, \frac{\partial}{\partial x_{j}} a_{j}, a_{0} \in L^{\infty}(\Omega)$ satisfying the conditions $a_{i j}(x)=a_{j i}(x)$ and

$$
\sum_{i, j=1}^{n} a_{i j}(x) \xi_{i} \xi_{j} \geq \delta_{0}|\xi|^{2} \quad \text { a.e. on } \Omega \text { for all } \xi \in \mathbb{R}^{n}
$$

with some $\delta_{0}>0$. Additionally, we require $a_{0}(x) \geq \delta_{1} \geq 0$ with $\delta_{1}$ sufficiently large such that the bilinear form $a(\cdot, \cdot)$ induced by $A$ fulfills the coercivity condition (1.1).

(iii) The obstacle $\psi$ fulfills $\psi \in H_{0}^{1}(\Omega) \cap H^{2}(\Omega)$ with $\max (0,-A \psi) \in L^{\infty}(\Omega)$.

As a consequence of assumption (iii) the bilinear form $a$ satisfies the weak maximum principle:

$$
a\left(y^{+}, y\right)=0 \text { implies that } y^{+}=0, \text { for } y \in H_{0}^{1}(\Omega),
$$

where $y^{+}=\max (0, y)$. Some of the results can be obtained under weaker regularity assumption on the domain $\Omega$ and the coefficients of $A$, specifically in these cases it suffices that the boundary of $\Omega$ is Lipschitz continuous and the coefficients of $A$ are sufficiently regular so that the bilinear form $a$ is well-defined on $H_{0}^{1}(\Omega) \times H_{0}^{1}(\Omega)$ and satisfies (1.1).

Let the functions $g, j$ satisfy:

(iv) $g: L^{2}(\Omega) \rightarrow \mathbb{R}$ is continuously Fréchet-differentiable and bounded from below, moreover the restriction $g: H_{0}^{1}(\Omega) \rightarrow \mathbb{R}$ is twice continuously Fréchet-differentiable.

(v) $j: L^{2}(\Omega) \rightarrow \mathbb{R}$ is twice continuously Fréchet-differentiable and weakly lower semi-continuous. Moreover, we assume $j$ to be radially unbounded, i.e. $j\left(u_{n}\right) \rightarrow+\infty$ whenever $\left\|u_{n}\right\|_{L^{2}(\Omega)} \rightarrow \infty$ with $u_{n} \in L^{2}(\Omega)$.

Some results can be obtained under weaker requirements on $g$.

Let us introduce the adjoint operator $A^{*}$ to $A$ by

$$
\left(A^{*} p\right)(x)=-\sum_{j=1}^{n} \frac{\partial}{\partial x_{j}}\left(\sum_{i=1}^{n} a_{i j}(x) \frac{\partial}{\partial x_{i}} p(x)+a_{j}(x) p(x)\right)+a_{0}(x) p(x) .
$$

Due to the assumptions on the coefficients, the equations $A y=f$ and $A^{*} p=g$ admit solutions in $H^{2}(\Omega)$ for right-hand sides $f, g \in L^{2}(\Omega)$.

Further assumptions will be introduced in the context of second order sufficient optimality conditions and well-posedness of the semi-smooth Newton method.

\section{NECESSARY AND SUFFICIENT OPTIMALITY CONDITIONS}

Let us briefly summarize known results about unique solvability of the underlying variational inequality (1.3).

Lemma 2.1. For each $u \in H^{-1}(\Omega)$ the variational inequality admits a unique solution $y \in H_{0}^{1}(\Omega)$, and the mapping $u \mapsto y$ is Lipschitz continuous from $u \in H^{-1}(\Omega)$ to $H_{0}^{1}(\Omega)$.

This lemma does not depend on the strong regularity assumptions (i)-(iii) above but the weaker ones mentioned below (iii) suffice. Employing the technique of [20], one obtains $L^{\infty}$-regularity of $y$. 
Lemma 2.2. For $u \in L^{2}(\Omega)$ the solution $y$ belongs to $L^{\infty}(\Omega)$, and the mapping $u \mapsto y$ is Lipschitz continuous from $u \in L^{2}(\Omega)$ to $L^{\infty}(\Omega)$.

Proof. Let $u_{1}, u_{2} \in L^{2}(\Omega)$ be given, and denote the associated solutions of the variational inequality by $y_{1}, y_{2}$. For $k \in \mathbb{R}, k \geq 0$ we introduce the truncation operator $[\cdot]_{k}: H_{0}^{1}(\Omega) \rightarrow H_{0}^{1}(\Omega)$ by

$$
\left([v]_{k}\right)(x)=\min (\max (v(x),-k), k) .
$$

Then the functions $v_{1}=y_{2}-\left[y_{2}-y_{1}\right]_{k}$ and $v_{2}=y_{1}+\left[y_{2}-y_{1}\right]_{k}$ are suitable as test functions for the variational inequality. In fact, if $y_{2}(x)-y_{1}(x)>k$ then $v_{1}(x)=y_{2}(x)-k \leq \psi(x)-k$ and $v_{2}(x)=y_{1}(x)+k<y_{2}(x)-k+k \leq$ $\psi(x)$. If $y_{2}(x)-y_{1}(x)<-k$ then $v_{1}(x)=y_{2}(x)+k<y_{1}(x)-k+k=y_{1}(x) \leq \psi(x)$ and $v_{2}(x)=y_{1}(x)-k \leq \psi(x)$, which altogether implies feasibility of $v_{1}, v_{2}$, i.e. $v_{1} \leq \psi$ and $v_{2} \leq \psi$.

Using $v_{1}$ and $v_{2}$ as test functions in the variational inequality for $y_{1}$ and $y_{2}$, respectively, and adding both inequalities, gives

$$
a\left(y_{1}-y_{2}, y_{1}-y_{2}-\left[y_{1}-y_{2}\right]_{k}\right) \leq\left(u_{1}-u_{2}, y_{1}-y_{2}-\left[y_{1}-y_{2}\right]_{k}\right) .
$$

With the notation $v:=y_{1}-y_{2}, v_{k}:=y_{1}-y_{2}-\left[y_{1}-y_{2}\right]_{k}$, we obtain by the properties of the differential operator

$$
a\left(v, v_{k}\right)=a\left(v_{k}, v_{k}\right) \leq\left(u_{1}-u_{2}, v_{k}\right) .
$$

Now, we can proceed as in Stampacchia [20], Theorem 4.1, to obtain the existence of a constant $c>0$ with

$$
\left\|y_{1}-y_{2}\right\|_{L^{\infty}}=\|v\|_{L^{\infty}} \leq c\left\|u_{1}-u_{2}\right\|_{L^{2}}
$$

where $c$ is independent of $u_{1}, u_{2}$.

Under the strong regularity assumptions above one has:

Lemma 2.3. For $u \in L^{2}(\Omega)$ the unique solution $(y, \lambda)$ of (1.3) belongs to $H_{0}^{1}(\Omega) \cap H^{2}(\Omega) \times L^{2}(\Omega)$. If in addition $u \in L^{p}(\Omega)$ and $\max (0, A \psi-u) \in L^{p}(\Omega)$, for $p \in[2, \infty)$, then $(y, \lambda) \in W^{2, p}(\Omega) \times L^{p}(\Omega)$.

Proof. The result can be obtained from Brezis and Stampacchia [4]. They proved under conditions which are implied by our regularity assumptions that $A y \in L^{p}(\Omega)$. Using elliptic regularity results $y \in W^{2, p}(\Omega)$ follows, see e.g. [8]. For a different approach we refer to [11].

Let us remark that the same regularity of solutions can be proven using the results of Grisvard [8] for convex and polygonal domains with $A$ the Laplace operator.

Lemma 2.4. The mapping $u \mapsto(y(u), \lambda(u))$ is directionally differentiable from $H^{-1}(\Omega)$ to $H_{0}^{1}(\Omega) \times H^{-1}(\Omega)$. The directional derivative $y^{\prime}(u ; h)=z$ satisfies $z \in S(y)$ and it is given as the solution of the variational inequality

where

$$
a(z, \phi-z) \geq(h, \phi-z) \text { for all } \phi \in S(y),
$$

$$
S(y)=\left\{\phi \in H_{0}^{1}(\Omega):\langle\lambda, \phi\rangle=0 \text {, and } \phi \leq 0 \text { whenever } y-\psi=0\right\} .
$$

Moreover, the directional derivative $\lambda^{\prime}(u ; h)=\eta \in H^{-1}(\Omega)$ satisfies

$$
A z+\eta=h,\langle\eta, y-\psi\rangle=0,\langle\eta, z\rangle=0
$$

and

$$
\langle\eta, \phi\rangle \leq 0, \quad \text { for all } \phi \in S(y) .
$$

Furthermore, we have for $\rho_{k} \in \mathbb{R}, \rho_{k}>0, \rho_{k} \rightarrow 0$, and $h_{k} \rightarrow h$ in $H^{-1}(\Omega)$

$$
\lim _{k \rightarrow \infty} \frac{1}{\rho_{k}}\left(\left\|y\left(u+\rho_{k} h_{k}\right)-y(u)-\rho_{k} y^{\prime}(u ; h)\right\|_{H^{1}}+\left\|\lambda\left(u+\rho_{k} h_{k}\right)-\lambda(u)-\rho_{k} \lambda^{\prime}(u ; h)\right\|_{H^{-1}}\right)=0 .
$$


This lemma again holds under the weaker regularity assumptions on $\Omega$ and the coefficients. Under the stronger assumptions, which imply $L^{2}-$ regularity of $\lambda$, the set $S(y)$ can equivalently be expressed as

$$
S(y)=\left\{\phi \in H_{0}^{1}(\Omega): \phi=0 \text { whenever } \lambda>0 \text { and } \phi \leq 0 \text { whenever } \lambda=y-\psi=0\right\} .
$$

Proof. Directional differentiability and the variational inequality $(2.1)$ for $y^{\prime}(u ; h)$ was proven by [15]. Let now two sequences $\rho_{k} \in \mathbb{R}, \rho_{k}>0, \rho_{k} \rightarrow 0$, and $h_{k} \rightarrow h$ in $H^{-1}(\Omega)$ be given. Let us write

$$
\begin{aligned}
\frac{1}{\rho_{k}}\left(y\left(u+\rho_{k} h_{k}\right)-y(u)\right)= & \frac{1}{\rho_{k}}\left(y\left(u+\rho_{k} h\right)-y(u)-\rho_{k} y^{\prime}(u ; h)\right) \\
& +\frac{1}{\rho_{k}}\left(y\left(u+\rho_{k} h_{k}\right)-y\left(u+\rho_{k} h\right)\right)+y^{\prime}(u ; h) .
\end{aligned}
$$

Due to Mignot's result, the first term on the right-hand side converges to zero in $H_{0}^{1}(\Omega)$ for $k \rightarrow \infty$. Since the mapping $u \mapsto y(u)$ is Lipschitz continuous, the second addend converges to zero in $H_{0}^{1}(\Omega)$, too. Due to the representation $\lambda=u-A y$, it follows that $u \mapsto \lambda(u)$ is directional differentiable from $H^{-1}(\Omega)$ to $H^{-1}(\Omega)$. Consequently we obtain (2.2).

It remains to prove the claimed properties of the directional derivatives. Setting $\eta=h-A z \in H^{-1}(\Omega)$ and testing

$$
\langle\eta, \phi-z\rangle \leq 0
$$

with $\phi=0, \phi=2 z$ and $\phi=z+\tilde{\phi}$, for $\tilde{\phi} \in S(y)$, we find $\langle\eta, z\rangle=0$ and $\langle\eta, \phi\rangle \leq 0$ for all $\phi \in S(y)$. Note that $\phi=0,2 z, z+\tilde{\phi}, \tilde{\phi} \in S(y)$, are suitable as test functions in (2.1).

To verify $\langle\eta, y-\psi\rangle=0$, we pass to the limit $t \searrow 0$ in

$$
\frac{1}{t}(\langle\lambda(u+t h), y(u+t h)-\psi\rangle-\langle\lambda(u), y(u)-\psi\rangle)=0
$$

as $t \rightarrow 0^{+}$using the product rule. Since $y^{\prime}(u ; h)=z \in S(y)$ we find $\langle\eta, y-\psi\rangle=0$ as desired.

If $\lambda \in L^{2}(\Omega)$ the lemma implies that pointwise a.e. $z \lambda=0$ is satisfied. If additionally the mapping $u \mapsto \lambda$ would be directionally differentiable with values in $L^{2}(\Omega)$, then using (1.5) for example, the following relations could be obtained on the biactive set $B$ :

$$
z \leq 0, \eta \geq 0, z \eta=0 \text { on } B=\{\lambda=0, y=\psi\} .
$$

Remark 2.5. If the biactive set $B$ is empty, then the mapping $u \mapsto y(u)$ is Gâteaux differentiable at $u$, see [15], and the system in Lemma 2.4 can be simplified as follows: The set $S(y)$ is now given by

$$
S(y)=\left\{\phi \in H_{0}^{1}(\Omega): \phi=0 \text { whenever } y-\psi=0\right\} .
$$

The directional derivative $z=y^{\prime}(u ; h) \in S(y)$ is given as the solution of the variational equation

$$
a(z, \phi)=(h, \phi) \text { for all } \phi \in S(y),
$$

which results in the following property of $\eta=\lambda^{\prime}(u ; h)$ :

$$
\langle\eta, \phi\rangle=0 \text { for all } \phi \in S(y) .
$$

Moreover, as proven in [15] the set of all $u \in L^{2}(\Omega)$ such that $y(\cdot)$ Gâteaux differentiable at $u$ is dense in $L^{2}(\Omega)$. 


\subsection{Necessary optimality condition}

Conditions (iv) and (v) of the standing assumptions together with Lemma 2.1 imply the existence of at least one solution $\left(y^{*}, u^{*}\right)$ with $y^{*}=y\left(u^{*}\right)$ to $(\mathrm{P})$.

Let us first state a consequence of local optimality, see e.g. [16] for a proof.

Lemma 2.6. Let $\left(y^{*}, u^{*}\right)$ be a locally optimal pair for the optimal control problem $(\mathrm{P})$. Then

$$
g^{\prime}\left(y^{*}\right) z+j^{\prime}\left(u^{*}\right) h \geq 0
$$

for all $h \in L^{2}(\Omega)$ and $z=y^{\prime}\left(u^{*} ; h\right)$.

The result of the previous lemma is a necessary optimality condition that is based solely on directional (Bouligand, conical) derivatives. In analogy to the terminology in mathematical programming with complementarity constraints (mpcc), we call this property B-stationarity. This stationarity result does not give any information about gradients and their representation by dual quantities. For this purpose we have the following result.

Theorem 2.7. Let $\left(y^{*}, u^{*}\right)$ be a locally optimal pair for the optimal control problem $(\mathrm{P})$ with associated multiplier $\lambda^{*} \in L^{2}(\Omega)$. Then there exist uniquely determined adjoint states $p^{*} \in H_{0}^{1}(\Omega) \cap L^{\infty}(\Omega)$ and $\mu^{*} \in$ $H^{-1}(\Omega) \cap\left(L^{\infty}(\Omega)\right)^{*}$ such that

$$
\begin{gathered}
A^{*} p^{*}+\mu^{*}+g^{\prime}\left(y^{*}\right)=0 \text { and } p^{*} \geq 0 \text { where } y^{*}=\psi, \\
\lambda^{*} p^{*}=0 \text { a.e. on } \Omega, \text { and }\left\langle\mu^{*}, p^{*}\right\rangle \geq 0 \\
\left\langle\mu^{*}, y^{*}-\psi\right\rangle=0, \\
\left\langle\mu^{*}, \phi\right\rangle \geq 0 \text { for all } \phi \in H_{0}^{1}(\Omega) \text { with }\left\langle\lambda^{*}, \phi\right\rangle=0 \text { and } \phi \geq 0 \text { on }\left\{y^{*}=\psi\right\}, \\
j^{\prime}\left(u^{*}\right)-p^{*}=0 .
\end{gathered}
$$

Moreover, we have the following sign condition for $\mu^{*}$ on B:

$$
\left\langle\mu^{*}, \phi\right\rangle \geq 0 \text { for all } \phi \in H_{0}^{1}(\Omega), \phi \geq 0 \text { on } B, \phi=0 \text { on } \Omega \backslash B \text {. }
$$

Proof. The existence and uniqueness of the adjoint state $p^{*}$ for given $\left(y^{*}, u^{*}\right)$ satisfying the following properties was proven for instance in $[15,16]$ :

$$
\begin{aligned}
& a\left(\phi, p^{*}\right)+\left(g^{\prime}\left(y^{*}\right), \phi\right) \leq 0 \\
& \text { for all } \phi \in H_{0}^{1}(\Omega) \text { with } \phi \geq 0 \text { on }\left\{y^{*}=\psi\right\} \text { and }\left\langle\lambda^{*}, \phi\right\rangle=0
\end{aligned}
$$

and

$$
p^{*} \geq 0 \text { on }\left\{y^{*}=\psi\right\},\left\langle\lambda^{*}, p^{*}\right\rangle=0, \text { and } j^{\prime}\left(u^{*}\right)=p^{*} .
$$

Setting $\mu^{*}=-A^{*} p^{*}-g^{\prime}\left(y^{*}\right)$ together with the previous properties implies (2.3) and (2.7). From (2.9) it follows that (2.6) is satisfied. Choosing $\phi \geq 0$ on $B$ and $\phi=0$ on $\Omega \backslash B$, (2.8) follows from (2.6). The property $\mu^{*} \in\left(L^{\infty}(\Omega)\right)^{*}$ was obtained in [12], Theorem 5.1.

From $\lambda^{*} \in L^{2}(\Omega)$ we conclude that $\lambda^{*} p^{*}=0$ a.e. on $\Omega$. In fact, $\lambda^{*}=0$ on $\left\{y^{*}<\psi\right\}$, and $\lambda^{*} \geq 0, p^{*} \geq 0$ on $\left\{y^{*}=\psi\right\}$ together with $\left\langle\lambda^{*}, p^{*}\right\rangle$ from (2.10) implies the claim.

Testing (2.9) with $p^{*}$ we have $\left\langle\mu^{*}, p^{*}\right\rangle \geq 0$, and thus (2.4) holds. Since $\psi=0$ on $\Gamma$, we can choose $\phi= \pm\left(y^{*}-\psi\right)$ in (2.6), which gives (2.5). 


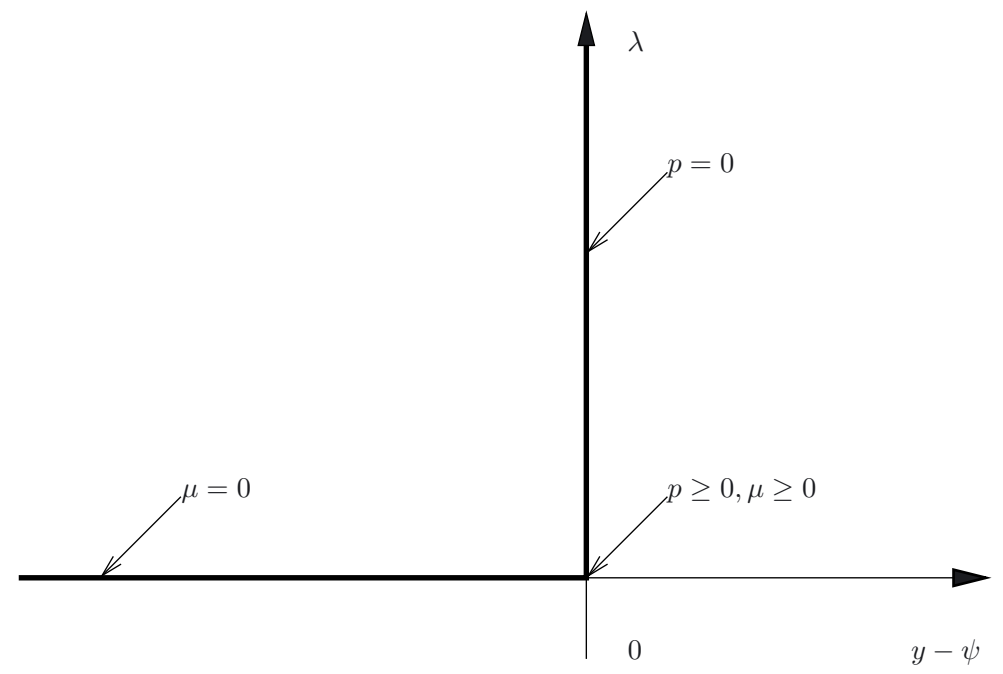

Figure 1. Complementarity relations.

The regularity $p^{*} \in L^{\infty}(\Omega)$ can be proven with arguments similar to the ones in [20] applied to the variational inequality. Here we use that $g^{\prime}\left(y^{*}\right) \in L^{2}(\Omega)$ and $\left(\lambda^{*},\left(p^{*}-k\right)^{+}\right)=0$ and $\left(\lambda^{*},\left(p^{*}+k\right)^{-}\right)=0$ for each $k \geq 0$, which holds since $p^{*} \lambda^{*}=0$. This implies that for positive $k$ the function

$$
p_{k}:= \begin{cases}p^{*}-k & \text { if } p^{*} \geq k \\ p^{*} & \text { if }\left|p^{*}\right|<k \\ p^{*}+k & \text { if } p^{*} \leq-k\end{cases}
$$

is suitable as test function in (2.9), which gives $a\left(p^{*}, p_{k}\right) \leq-\left(g^{\prime}(y), p_{k}\right)$. Due to the smoothness assumptions on the coefficients in the differential operator, there exists $\bar{\sigma} \geq 0$ such that $\bar{\sigma}+a_{0}-\sum_{i=1}^{n} \frac{\partial}{\partial x_{i}} a_{j} \geq 0$ a.e. on $\Omega$. Then integration by parts yields $a\left(p^{*}, p_{k}\right)+\bar{\sigma}\left(p^{*}, p_{k}\right) \geq a\left(p_{k}, p_{k}\right)+\bar{\sigma}\left(p_{k}, p_{k}\right)$, and we can proceed exactly as in the proof of [20], Theorem 4.1, to obtain $p^{*} \in L^{\infty}(\Omega)$.

Figure 1 illustrates the relationship of primal and dual variables on the active, bi-active and inactive sets.

Under special assumptions on the function $j$, one can conclude higher regularity of the optimal controls.

Corollary 2.8. Let $j(u)=\frac{\alpha}{2}\|u\|_{L^{2}}^{2}, \alpha>0, \psi \in W^{2, p}(\Omega), p>2$, and let $\left(y^{*}, u^{*}\right)$ be locally optimal. Then $u^{*} \in H^{1}(\Omega) \cap L^{\infty}(\Omega)$ and $y^{*} \in W^{2, p}(\Omega)$.

Proof. From (2.7) of Theorem 2.7 it follows that $u^{*} \in L^{\infty}(\Omega)$. The regularity result for $y^{*}$ is a consequence of Lemma 2.3.

If the control-to-state mapping $u \mapsto y(u)$ is Gâteaux-differentiable, see Remark 2.5, then the optimality system can be rephrased as follows, see [15], p. 161.

Corollary 2.9. Let the assumptions of Theorem 2.7 be satisfied. Let $y(\cdot)$ be Gâteaux differentiable at $u^{*}$. Then we have

$$
a\left(\phi, p^{*}\right)+\left(g^{\prime}\left(y^{*}\right), \phi\right)=0 \quad \text { for all } \phi \in S(y) .
$$

In particular, the relations $\left\langle\eta, p^{*}\right\rangle=\left\langle\mu^{*}, z\right\rangle=0$ for $z=y^{\prime}\left(u^{*} ; h\right), \eta=\lambda^{\prime}\left(u^{*} ; h\right), h \in L^{2}(\Omega)$ hold.

As already mentioned, optimal control of variational inequalities can be interpreted as optimization with complementarity constraints. In analogy to the analysis of mpcc problems in finite dimensions, the system (2.3)-(2.8) 
describes strong stationarity, see also [9]. Without the information on the sign of $p^{*}$ and $\mu^{*}$ on the biactive set, the system (2.3)-(2.7) denotes C-stationarity, which is a weaker form of stationarity. For a survey of different stationarity concepts for finite-dimensional optimization problems with complementarity constraints we refer to $[19]$.

If one would formally derive the optimality system using the Lagrangian

$$
L(y, u, \lambda, p, \mu, q)=g(y)+j(u)+\langle A y+\lambda-u, p\rangle+\langle\mu, y-\psi\rangle+\langle\lambda, q\rangle
$$

then the adjoint state $p$ could be identified with the multiplier $q$ of the constraint $\lambda \geq 0$. The measure $\mu$ would play the role of a multiplier to the state constraint $y \leq \psi$. The constraint $\langle\lambda, y-\psi\rangle=0$ is treated implicitly, since the existence of a Lagrange multiplier to $\langle\lambda, y-\psi\rangle=0$ does not follow from first-order optimality conditions, see the discussion and counterexample in [3].

\section{Remark 2.10.}

(i) In the case of strict complementarity, i.e. $\lambda^{*}>0$ on $y^{*}=\psi$, we have $p^{*}=0$ on the active set $\left\{\psi=y^{*}\right\}$ by $(2.3)$ and (2.4).

(ii) The support of $\mu^{*}$ is contained on the active set $\left\{y^{*}=\psi\right\}$ : In fact, by (2.6) we have $\left\langle\mu^{*}, \phi\right\rangle=0$ for all $\phi \in H_{0}^{1}(\Omega)$ with $\phi=0$ on the active set. Moreover, (2.8) gives information on the sign of $\mu$ on the bi-active set.

Since we have $\mu^{*} \in\left(L^{\infty}(\Omega)\right)^{*}$, we can write $\mu^{*}=\mu_{r}+\mu_{p}$, where $\mu_{r} \in L^{1}(\Omega)$ is the regular part of $\mu^{*}$, and $\mu_{p}$ is purely finitely additive in the sense of Yosida and Hewitt [22]. The addends $\mu_{r}$ and $\mu_{p}$ are uniquely determined [22], Theorem 1.24. Regarding the structure of $\mu$ the following result holds.

Proposition 2.11. Under the assumptions of Theorem 2.7 we have

$$
\begin{aligned}
\operatorname{supp} \mu_{p} & \subset \overline{\left\{\lambda^{*}=0\right\}} \cap \overline{\left\{y^{*}=\psi\right\}} \backslash \Gamma \\
\mu_{r} & =-\chi_{\left\{\lambda^{*}>0\right\}} g^{\prime}\left(y^{*}\right) .
\end{aligned}
$$

In the case of strict complementarity $(B=\emptyset)$ we find

$$
\operatorname{supp} \mu_{p} \subset \partial\left\{y^{*}=\psi\right\} \backslash \Gamma=\partial\left\{\lambda^{*}=0\right\} \backslash \Gamma .
$$

Proof. Take $\phi \in H_{0}^{1}(\Omega) \cap L^{\infty}(\Omega)$ with $\phi=0$ on $\left\{\lambda^{*}=0\right\}$. Then we have $\phi p^{*}=0$ a.e. on $\Omega$, which gives $a\left(\phi, p^{*}\right)=0$. Utilizing $\phi$ as test function in the adjoint equation (2.3) implies

$$
\left\langle\mu^{*}, \phi\right\rangle=\left\langle\mu_{p}, \phi\right\rangle+\int_{\left\{\lambda^{*}>0\right\}} \mu_{r} \phi=-\int_{\left\{\lambda^{*}>0\right\}} g^{\prime}\left(y^{*}\right) \phi .
$$

Therefore $\left\langle\mu_{p}, \phi\right\rangle=0$ and $\mu_{r}=-g^{\prime}\left(y^{*}\right)$ on $\left\{\lambda^{*}>0\right\}$. Moreover, we obtain from Theorem 2.7, (2.6) that $\left\langle\mu^{*}, \tilde{\phi}\right\rangle=0$ for all regular $\tilde{\phi}$ with $\tilde{\phi}=0$ on $\left\{y^{*}=\psi\right\}$, which gives $\left\langle\mu_{p}, \tilde{\phi}\right\rangle=0$. Hence, the support of $\mu_{p}$ is contained in $\overline{\left\{\lambda^{*}=0\right\}} \cap \overline{\left\{y^{*}=\psi\right\}} \backslash \Gamma$.

In general, $\partial\left\{y^{*}=\psi\right\}$ and $\partial\left\{\lambda^{*}=0\right\}$ are subsets of $\overline{\left\{\lambda^{*}=0\right\}} \cap \overline{\left\{y^{*}=\psi\right\}}$. In the case $B=\emptyset$ the reverse inclusion can be proven, and the claim follows.

If in addition $y^{*}, \psi, \lambda^{*}$ would be continuous, then supp $\mu_{p} \subset B$ could be obtained.

\subsection{Sufficient optimality condition}

Now let us introduce the coercivity condition that will ensure local optimality. 


\section{Assumption 1.}

(i) There is $\gamma>0$ such that

$$
j^{\prime \prime}\left(u^{*}\right)(h, h) \geq \gamma\|h\|_{L^{2}}^{2} \quad \text { for all } h \in L^{2}(\Omega) .
$$

(ii) For all $h \in L^{2}(\Omega) \backslash\{0\}$ and $z=y^{\prime}\left(u^{*} ; h\right)$ with $j^{\prime}\left(u^{*}\right) h+g^{\prime}\left(y^{*}\right) z=0$, we have

$$
g^{\prime \prime}\left(y^{*}\right)(z, z)+j^{\prime \prime}\left(u^{*}\right)(h, h)>0 .
$$

(iii) There exists a constant $\tau>0$ such that

$$
p^{*} \geq 0 \text { on }\left\{\psi-\tau<y^{*}<\psi\right\} .
$$

(iv) Moreover, $\mu^{*}$ satisfies

$$
\mu^{*} \geq 0
$$

The following theorem asserts that the first order optimality condition given by Theorem 2.7 together with Assumption 1 imply local optimality.

Theorem 2.12. Let $\left(y^{*}, u^{*}, \lambda^{*}, p^{*}, \mu^{*}\right)$ satisfy the first-order optimality system (2.3)-(2.7) given by Theorem 2.7 . If Assumption 1 is fulfilled, then $\left(y^{*}, u^{*}\right)$ is locally optimal, and there exist $\alpha>0, r>0$ such that

$$
\begin{aligned}
& J\left(y^{*}, u^{*}\right)+\alpha\left\|v-u^{*}\right\|_{L^{2}}^{2} \leq J(y(v), v) \\
& \text { for all } v \in L^{2}(\Omega) \text { with }\left\|u^{*}-v\right\|_{L^{2}}<r .
\end{aligned}
$$

Before we enter into the proof of this theorem we address Assumption 1.

\section{Remark 2.13.}

(i) Assumption 1 (i) and (ii) are trivially fulfilled for the quadratic functional

$$
J(y, u)=\frac{1}{2}\left\|y-y_{d}\right\|_{L^{2}}^{2}+\frac{\alpha}{2}\left\|u-u_{d}\right\|_{L^{2}}^{2}
$$

with $\alpha>0, y_{d} \in L^{2}(\Omega)$ and $u_{d} \in L^{2}(\Omega)$. If, moreover

$$
\psi \leq y_{d}
$$

then the mapping $u \rightarrow \frac{1}{2}\left\|y(u)-y_{d}\right\|_{L^{2}}^{2}$ is convex, see [15], Theorem 4.1, and hence $u \rightarrow J(y(u), u)$ is strictly convex. Inequality (2.17) is also sufficient for Assumption 1 to hold in the case of the quadratic cost (2.16), as we will discuss next.

(ii) Concerning Assumption 1 (iii) let us assume that

$$
g^{\prime}\left(y^{*}\right) \leq 0 .
$$

This inequality is satisfied for the quadratic cost (2.16) if $y^{*} \leq y_{d}$ which, in turn is implied by $\psi \leq y_{d}$.

With $g^{\prime}\left(y^{*}\right) \leq 0$ holding let us decompose $p^{*}$ as $\left(p^{*}\right)^{+}-\left(p^{*}\right)^{-}$with $\left(p^{*}\right)^{+}=\max \left(0, p^{*}\right)$ and $\left(p^{*}\right)^{-}=$ $\max \left(0,-p^{*}\right)$. Testing the adjoint equation $(2.3)$ with $\left(p^{*}\right)^{-}$, we find

$$
-a\left(\left(p^{*}\right)^{-}, p^{*}\right)-\left\langle\mu^{*},\left(p^{*}\right)^{-}\right\rangle=\left(g^{\prime}\left(y^{*}\right),\left(p^{*}\right)^{-}\right) \leq 0 .
$$

By (2.3) we have $\left(p^{*}\right)^{-}=0$ on $\left\{x: y^{*}(x)=\psi(x)\right\}$, from (2.4) we deduce $\lambda^{*}\left(p^{*}\right)^{-}=0$ a.e. in $\Omega$ and hence $\left\langle\lambda^{*},\left(p^{*}\right)^{-}\right\rangle=0$. In particular, both $\left(p^{*}\right)^{-}$and $-\left(p^{*}\right)^{-}$serve as test functions $\theta$ in (2.6). Consequently (2.6) implies that $\left\langle\mu^{*},\left(p^{*}\right)^{-}\right\rangle=0$. We obtain

$$
-a\left(\left(p^{*}\right)^{-}, p^{*}\right)=\left(g^{\prime}\left(y^{*}\right),\left(p^{*}\right)^{-}\right) \leq 0,
$$

thus by the standing assumptions $\left(p^{*}\right)^{-}=0$ is satisfied, and Assumption 1 (iii) holds. 
(iii) Next we discuss two cases in which Assumption 1 (iv) can be guaranteed.

(1) Consider the approximating system (1.7) with multiplier $\mu_{c}$ defined as $\mu_{c}=c \operatorname{sgn}_{c}(\bar{\lambda}+$ $\left.c\left(y_{c}-\psi\right)\right) p_{c}$, and note that by the weak maximum principle

$$
p_{c} \geq 0 \text { and } \mu_{c} \geq 0 \text { if } g^{\prime}\left(y_{c}\right) \leq 0 .
$$

If $\left(y_{c}, u_{c}\right)$ are global solutions to $\left(P_{c}\right)$ then sufficient conditions for convergence of $\mu_{c}$ to $\mu^{*} \in L^{\infty}(\Omega)^{*} \cap$ $H_{0}^{1}(\Omega)$ will be given in Proposition 3.8. Then (2.19) implies Assumption 1 (iv). The condition $g^{\prime}\left(y_{c}\right) \leq 0$ is satisfied in the feasible case for the quadratic cost (2.16) if (2.17) holds. In fact, in this case $g^{\prime}\left(y_{c}\right)=y_{c}-y_{d} \leq \psi-y_{d} \leq 0$.

In particular for the quadratic cost (2.16) with $\psi \leq y_{d}$ Assumption 1 is satisfied.

(2) For the second case we consider, for simplicity of representation, $A=-\Delta$. We focus on a local solution $\left(y^{*}, u^{*}\right)$ of $(P)$, and make the structural assumptions of strict complementarity an that the active set $\mathcal{A}^{*}=\left\{x \in \Omega: y^{*}(x)=\psi(x)\right\}$ consists of a connected component satisfying $\overline{\operatorname{int} \mathcal{A}^{*}}=\mathcal{A}^{*} \subset \Omega$. We set $\mathcal{I}^{*}=\Omega \backslash \mathcal{A}^{*}$, and assume that $\partial \mathcal{A}^{*}$ is $C^{1,1}$ regular. The situation when $\mathcal{A}^{*}$ consists of several connected components or of an interior arc can be treated with similar techniques. We consider the very weak form of (2.3) given by

$$
-\left(p^{*}, \Delta \phi\right)_{\Omega}+\left\langle\mu^{*}, \phi\right\rangle+\left(g^{\prime}\left(y^{*}\right), \phi\right)_{\Omega}=0, \text { for all } \phi \in H_{0}^{1}(\Omega),
$$

and successively consider test functions with compact support in $\mathcal{A}^{*}, \mathcal{I}^{*}$ and $\Omega$, keeping in mind from Remark 2.10 that $p^{*}=0$ on $\mathcal{A}^{*}$ and that the support of $\mu^{*}=\mu_{r}^{*}+\mu_{p}^{*}$ is contained in $\mathcal{A}^{*}$. Then we obtain, with a computation very similar to the one for the proof of Theorem 2 in [2]

$$
\begin{cases}A p^{*}=-g^{\prime}\left(y^{*}\right) & \text { in } \mathcal{I}^{*} \\ p^{*}=0 & \text { on } \partial \Omega \cup \partial \mathcal{A}^{*},\end{cases}
$$

and

$$
\left\{\begin{aligned}
\mu_{r}^{*} & =-g^{\prime}\left(y^{*}\right) \text { on } \mathcal{A}^{*} \\
-\frac{\partial p^{*}}{\partial n_{\mathcal{I}}} & =\mu_{p}^{*}
\end{aligned}\right.
$$

where $n_{\mathcal{I}}$ is the exterior normal to $\mathcal{I}^{*}$. If $g^{\prime}\left(y^{*}\right) \leq 0$ a.e. in $\Omega$, then $\mu_{r}^{*} \geq 0$ in $\mathcal{A}^{*}$. Moreover, in this case $p^{*} \geq 0$ a.e. in $\mathcal{I}^{*}$, and $\frac{\partial p^{*}}{\partial n_{\mathcal{I}}} \leq 0$ on $\partial \mathcal{A}^{*}$, since $p^{*}=0$ on $\mathcal{A}^{*}$. Hence $\mu_{r}^{*} \geq 0$ and thus $\mu^{*} \geq 0$ and Assumption 1 (iv) is satisfied.

(iv) Assumption 1 can be satisfied without $\psi \leq y_{d}$ holding. For this purpose consider the functional (2.16), and set $\Omega=(0,1), a(v, w)=\int_{0}^{1} v^{\prime} w^{\prime} \mathrm{d} x$, and define the functions

$$
\left\{\begin{array}{l}
y=p=\frac{x}{2}(1-x) \geq 0, \quad \lambda=\mu=0, \\
\psi=y \text { on }\left(0, \frac{1}{4}\right) \cup\left(\frac{3}{4}, 1\right), \psi \in H_{0}^{1}, \text { and } \psi \text { arbitrarily large on }\left(\frac{1}{4}, \frac{3}{4}\right), \\
u=\frac{1}{2}, \quad y_{d}=\frac{1}{2}+\frac{x}{2}(1-x), \\
u_{d}=u-p=\frac{1}{2}+\frac{x}{2}(x-1)
\end{array}\right.
$$

For this choice of $y, p, u, \lambda, \mu, y_{d}, u_{d}$ the necessary optimality conditions of Theorem 2.7, as well as Assumption 1 are satisfied. On the other hand $\psi \leq y_{d}$ does not hold.

(v) For the choice

$$
g(y)=\int_{\Omega} \operatorname{arccot} y \mathrm{~d} x
$$

the regularity requirements (iv) of the standing assumptions as well as the sign conditions in Assumption 1 (iii) and (iv) are satisfied. In fact, refering to [21], Chapter 3, the mapping $y \rightarrow g(y)=$ $\int_{\Omega} \operatorname{arccot} y \mathrm{~d} x$ is in $C^{1}\left(L^{2}(\Omega), \mathbb{R}\right) \cap C^{2}\left(H_{0}^{1}(\Omega), \mathbb{R}\right)$. Clearly $g^{\prime}(y) \leq 0$ for any $y \in H_{0}^{1}(0)$ and hence 
$p^{*} \geq 0$ by (ii) of this remark. Moreover by (2.19) we have $p_{c} \geq 0$ and $\mu_{c} \geq 0$. By (iii) of this remark we get $\mu^{*} \geq 0$.

(vi) The sign conditions on $\mu^{*}$ and on $p^{*}$ on the almost bi-active set $\left\{\psi-\tau<y^{*}<\psi\right\} \cap\{\lambda=0\}$, constitute the major difference in comparison to second-order sufficient optimality conditions for stateconstrained optimal control problems and to finite-dimensional mpcc's. For state constrained problems, sign conditions like (2.13) and (2.14) are not necessary, since there the non-negativity of multipliers follows from the first-order necessary optimality conditions. This is not the case here. For example, the sign of $p^{*}$ is unknown on the inactive set $\left\{y^{*}<\psi\right\}$ as is the sign of $\mu^{*}$ on the active set, see also Figure 1.

In the finite-dimensional case, one can find a neighborhood $\mathcal{U}$ of $u^{*}$ such that inactive constraints $y^{*}<\psi$ or strongly active constraints $\lambda^{*}>0$ remain inactive or strongly active, respectively. Hence, the sign information on the multipliers $p^{*}$ and $\mu^{*}$ is enough, and it holds $\left\langle\lambda(u)-\lambda^{*}, p^{*}\right\rangle \geq 0$ and $-\left\langle\mu^{*}, y(u)-y^{*}\right\rangle \geq 0$ for all $u \in \mathcal{U}$, see $(2.26)$ and (2.27) below. This does not work for the infinitedimensional problem considered here, since the active set may change with any small perturbation of the control $u^{*}$.

Proof of Theorem 2.12. Let us assume that the quadratic growth condition (2.15) does not hold. Then there exist sequences $u_{k}, u_{k} \rightarrow u^{*}$ in $L^{2}(\Omega), y_{k}=y\left(u_{k}\right), \lambda_{k}=\lambda\left(u_{k}\right)$, such that

$$
J\left(y^{*}, u^{*}\right)+\frac{1}{k}\left\|u_{k}-u^{*}\right\|_{L^{2}}^{2}>J\left(y_{k}, u_{k}\right) .
$$

Introducing the quantities $\rho_{k}=\left\|u_{k}-u^{*}\right\|_{L^{2}}$ and $h_{k}:=\frac{1}{\rho_{k}}\left(u_{k}-u^{*}\right)$, this inequality becomes

$$
J\left(y^{*}, u^{*}\right)+\frac{\rho_{k}^{2}}{k}>J\left(y_{k}, u_{k}\right) .
$$

By construction $\left\|h_{k}\right\|_{L^{2}(\Omega)}=1$ and by Lemma 2.1 the sequence $z_{k}:=\frac{1}{\rho_{k}}\left(y_{k}-y^{*}\right)$ is bounded in $H_{0}^{1}(\Omega)$. Moreover the sequence $\eta_{k}:=\frac{1}{\rho_{k}}\left(\lambda_{k}-\lambda^{*}\right)$ is bounded in $H^{-1}(\Omega)$. This is a consequence of (1.3), which implies that

$$
\eta_{k}=\frac{1}{\rho_{k}}\left(\lambda_{k}-\lambda^{*}\right)=\frac{1}{\rho_{k}}\left(u_{k}-u^{*}-A\left(y_{k}-y^{*}\right)\right)=h_{k}-A z_{k} .
$$

Thus we have by compact embeddings and after extracting subsequences

$$
h_{k} \rightarrow h \text { in } L^{2}(\Omega), \quad h_{k} \rightarrow h \text { in } H^{-1}(\Omega) .
$$

Due to Lemma 2.4, equation (2.2), we obtain the strong convergence

$$
z_{k} \rightarrow z=y^{\prime}\left(u^{*} ; h\right) \text { in } H_{0}^{1}(\Omega), \quad \eta_{k} \rightarrow \eta=\lambda^{\prime}\left(u^{*} ; h\right) \text { in } H^{-1}(\Omega) .
$$

Let us investigate the difference of the values of the objective functional $J$. Using the optimality system, we find

$$
\begin{aligned}
J\left(y_{k}, u_{k}\right)-J\left(y^{*}, u^{*}\right)= & g^{\prime}\left(y^{*}\right)\left(y_{k}-y^{*}\right)+\frac{1}{2} g^{\prime \prime}\left(\tilde{y}_{k}\right)\left(y^{*}-y_{k}\right)^{2} \\
& +j^{\prime}\left(u^{*}\right)\left(u_{k}-u^{*}\right)+\frac{1}{2} j^{\prime \prime}\left(\tilde{u}_{k}\right)\left(u^{*}-u_{k}\right)^{2} \\
& +\left\langle A\left(y_{k}-y^{*}\right)+\lambda_{k}-\lambda^{*}-\left(u_{k}-u^{*}\right), p^{*}\right\rangle \\
= & \frac{1}{2} g^{\prime \prime}\left(\tilde{y}_{k}\right)\left(y^{*}-y_{k}\right)^{2}+\frac{1}{2} j^{\prime \prime}\left(\tilde{u}_{k}\right)\left(u^{*}-u_{k}\right)^{2} \\
& +\left\langle\lambda_{k}-\lambda^{*}, p^{*}\right\rangle-\left\langle\mu^{*}, y_{k}-y^{*}\right\rangle<\frac{\rho_{k}^{2}}{k},
\end{aligned}
$$


where $\tilde{y}_{k}$ and $\tilde{u}_{k}$ denote elements between $y_{k}$ and $y^{*}$, and $u_{k}$ and $u^{*}$, respectively. We obtain

$$
\begin{aligned}
\left\langle\frac{1}{\rho_{k}}\left(\lambda_{k}-\lambda^{*}\right), p^{*}\right\rangle-\left\langle\mu^{*}, \frac{1}{\rho_{k}}\left(y_{k}-y^{*}\right)\right. & \\
& <\frac{\rho_{k}}{k}-\frac{\rho_{k}}{2}\left(g^{\prime \prime}\left(\tilde{y}_{k}\right)\left(\frac{1}{\rho_{k}}\left(y^{*}-y_{k}\right)\right)^{2}+j^{\prime \prime}\left(\tilde{u}_{k}\right)\left(\frac{1}{\rho_{k}}\left(u^{*}-u_{k}\right)\right)^{2}\right),
\end{aligned}
$$

or with the notation introduced above

$$
\left\langle\eta_{k}, p^{*}\right\rangle-\left\langle\mu^{*}, z_{k}\right\rangle<\frac{\rho_{k}}{k}-\frac{\rho_{k}}{2}\left(g^{\prime \prime}\left(\tilde{y}_{k}\right)\left(z_{k}, z_{k}\right)+j^{\prime \prime}\left(\tilde{u}_{k}\right)\left(h_{k}, h_{k}\right)\right) .
$$

Together with (2.21), the general assumptions (iv) and (v), and the fact that $\lim _{k \rightarrow \infty} \rho_{k}=0$ this implies

$$
\left\langle\eta, p^{*}\right\rangle-\left\langle\mu^{*}, z\right\rangle \leq 0
$$

Since $A z+\eta=h$ equations (2.3) and (2.7) of the first order optimality system imply the identity

$$
\left\langle\eta, p^{*}\right\rangle-\left\langle\mu^{*}, z\right\rangle=j^{\prime}\left(u^{*}\right) h+g^{\prime}\left(y^{*}\right) z .
$$

By B-stationarity, cf. Lemma 2.6, we find that $j^{\prime}\left(u^{*}\right) h+g^{\prime}\left(y^{*}\right) z \geq 0$, which in turn gives $\left\langle\eta, p^{*}\right\rangle-\left\langle\mu^{*}, z\right\rangle \geq 0$, and hence $\left\langle\eta, p^{*}\right\rangle-\left\langle\mu^{*}, z\right\rangle=0$, which, in turn implies that

$$
j^{\prime}\left(u^{*}\right) h+g^{\prime}\left(y^{*}\right) z=0 .
$$

Now, let us prove that the left-hand side of (2.22) is non-negative for $k$ large enough. Due to Lemma 2.2 we can choose $k_{0}$ sufficiently large, such that $\left\|y^{*}-y_{k}\right\|_{L^{\infty}}<\tau$ for all $k>k_{0}$, where $\tau$ is chosen according to Assumption 1 (iii). Then the inclusions supp $\lambda_{k} \subset\left\{y_{k}=\psi\right\} \subset\left\{\psi-\tau<y^{*} \leq \psi\right\}$ hold. We obtain using $\lambda^{*} p^{*}=0$ and $p^{*} \geq 0$ on $\left\{\psi-\tau<y^{*} \leq \psi\right\}$, see (2.3), (2.13),

$$
\left\langle\lambda_{k}-\lambda^{*}, p^{*}\right\rangle=\int_{\psi-\tau<y^{*} \leq \psi} \lambda_{k} p^{*} \geq 0 .
$$

Since $\left\langle\mu^{*}, y^{*}-\psi\right\rangle=0$ by $(2.5)$, we have as a consequence of (2.14)

$$
-\left\langle\mu^{*}, y_{k}-y^{*}\right\rangle=-\left\langle\mu^{*}, y_{k}-\psi\right\rangle \geq 0
$$

for all $k$. Combining (2.22)-(2.27), we obtain

$\frac{1}{2}\left(g^{\prime \prime}\left(y^{*}\right)\left(z_{k}, z_{k}\right)+j^{\prime \prime}\left(u^{*}\right)\left(h_{k}, h_{k}\right)\right)<\frac{1}{k}-\frac{1}{2}\left(g^{\prime \prime}\left(\tilde{y}_{k}\right)\left(z_{k}, z_{k}\right)-g^{\prime \prime}\left(y^{*}\right)\left(z_{k}, z_{k}\right)+j^{\prime \prime}\left(\tilde{u}_{k}\right)\left(h_{k}, h_{k}\right)-j^{\prime \prime}\left(u^{*}\right)\left(h_{k}, h_{k}\right)\right)$.

Since $j^{\prime \prime}$ and $g^{\prime \prime}$ are continuous by assumption, and the sequences $h_{k}$ and $z_{k}$ are bounded, the right-hand side vanishes for $k \rightarrow \infty$. Turning to the left hand side, we recall that $z_{k} \rightarrow z$ in $H_{0}^{1}(\Omega)$ strongly by (2.21). The positivity requirement Assumption 1 (i) on $j^{\prime \prime}$, implies that $j^{\prime \prime}\left(u^{*}\right)(h, h) \leq \liminf j^{\prime \prime}\left(u^{*}\right)\left(h_{k}, h_{k}\right)$. Consequently

$$
g^{\prime \prime}\left(y^{*}\right)(z, z)+j^{\prime \prime}\left(u^{*}\right)(h, h) \leq 0 .
$$

By Assumption 1 (ii), which is applicable due to (2.25), we find $h=0$. This in turn gives $z=0$, since the variational inequality (2.1) determining $z$ is uniquely solvable. Due to $\left\|h_{k}\right\|_{L^{2}}=1$ and Assumption 1 (i) we have

$$
0<\frac{\gamma}{2} \leq \frac{1}{2} j^{\prime \prime}\left(u^{*}\right)\left(h_{k}, h_{k}\right)<\frac{1}{k}-\frac{1}{2}\left(g^{\prime \prime}\left(\tilde{y}_{k}\right)\left(z_{k}, z_{k}\right)+j^{\prime \prime}\left(\tilde{u}_{k}\right)\left(h_{k}, h_{k}\right)-j^{\prime \prime}\left(u^{*}\right)\left(h_{k}, h_{k}\right)\right) .
$$

Since $z_{k} \rightarrow z=0$ strongly in $H_{0}^{1}(\Omega)$, the right-hand side vanishes for $k \rightarrow \infty$ yielding finally the contradiction. 
We end this section with a technical remark regarding the previous proof.

\section{Remark 2.14.}

(i) If one would have strong convergence $\lambda_{k} \rightarrow \lambda^{*}$ in $L^{\infty}(\Omega)$, then one could weaken the non-negativity assumption on $\mu^{*}=\mu_{p}+\mu_{r}$ to: $\mu_{p} \geq 0$ and $\mu_{r} \geq 0$ on $0<\lambda^{*}<\tau$, and the corresponding arguments are analogous to (2.26) to obtain the conclusion of Theorem 2.12 .

(ii) If the mapping $y(\cdot)$ is Gâteaux differentiable at $u^{*}$, the condition $j^{\prime}\left(u^{*}\right) h+g^{\prime}\left(y^{*}\right) z=0$ in Assumption 1 (ii) is redundant. In fact, then one has by (2.24) and Corollary 2.9

$$
j^{\prime}\left(u^{*}\right) h+g^{\prime}\left(y^{*}\right) z=\left\langle\eta, p^{*}\right\rangle-\left\langle\mu^{*}, z\right\rangle=0 .
$$

\section{Convergence Properties AND FEASIBILITy of REGUlarized PROBlems}

For numerical purposes the optimality system obtained in Theorem 2.7 has the disadvantage of involving Lagrange multipliers that are measures. This is one of the motivations to introduce regularisation techniques, which we already announced in Section 1 in form of problem $\left(\mathrm{P}_{c}\right)$.

\subsection{Convergence properties for the regularized problems}

This subsection is devoted to a brief summary of the convergence properties of the regularized problems $\left(\mathrm{P}_{c}\right)$ as $c \rightarrow \infty$. Let us commence with considering the regularized equation

$$
A y+\max _{c}(0, \bar{\lambda}+c(y-\psi))=u,
$$

and define

$$
\lambda_{c}=\max _{c}(0, \bar{\lambda}+c(y-\psi)) .
$$

Throughout this and the following section, we require that $\bar{\lambda} \in L^{\infty}(\Omega)$ is a non-negative function, which is fixed during the regularization process $c \rightarrow \infty$.

The following convergence result is taken from [13].

Lemma 3.1. Let $\bar{\lambda} \in L^{\infty}(\Omega)$ be given. For $u \in L^{2}(\Omega)$ let $\left(y_{c}, \lambda_{c}\right)$ denote the solution to (3.1). Then $\left(y_{c}, \lambda_{c}\right)$ converge to the unique solution $(y, \lambda)$ of $(1.3)$ in the sense that $y_{c} \rightarrow y=y(u)$ in $H_{0}^{1}(\Omega)$ and $\lambda_{c} \rightarrow \lambda$ in $H^{-1}(\Omega)$ strongly as $c \rightarrow \infty$.

In addition, we obtain convergence rate results in $L^{\infty}(\Omega)$.

Lemma 3.2. Let $\bar{\lambda} \in L^{\infty}(\Omega)$ be given. Then for each $c>0$

$$
y \leq y_{c}+\frac{\|\bar{\lambda}\|_{L^{\infty}}}{c}+\frac{1}{2 c^{2}} \quad \text { a.e. on } \Omega .
$$

Proof. Let us define for $k \geq 0$ the function $\phi_{k}=\max \left(y-y_{c}-k, 0\right) \in H_{0}^{1}(\Omega)$. Subtracting (3.1) from the first equation in (1.3) and testing with $\phi_{k}$ gives

$$
a\left(y-y_{c}-k, \phi_{k}\right)+\left\langle\lambda-\max _{c}\left(0, \bar{\lambda}+c\left(y_{c}-\psi\right)\right), \phi_{k}\right\rangle+a\left(k, \phi_{k}\right)=0 .
$$

Let us set $k:=\frac{\|\bar{\lambda}\|_{L} \infty}{c}+\frac{1}{2 c^{2}}$. Since $\lambda \geq 0$, we have $\left\langle\lambda, \phi_{k}\right\rangle \geq 0$. Moreover, since $y \leq \psi$ we obtain on the set $\left\{\phi_{k}>0\right\}$

$$
\begin{aligned}
\max _{c}\left(0, \bar{\lambda}+c\left(y_{c}-\psi\right)\right) & \leq \max _{c}\left(0, \bar{\lambda}+c\left(y_{c}-y\right)\right) \\
& \leq \max _{c}(0, \bar{\lambda}-c k) \leq \max _{c}\left(0,-\frac{1}{2 c}\right)=0
\end{aligned}
$$


which, together with $a\left(1, \phi_{k}\right) \geq 0$ implies that $a\left(y-y_{c}-k, \phi_{k}\right) \leq 0$, and hence $\phi_{k}=0$, which implies the desired result.

Remark 3.3. In [13] a bilateral $L^{\infty}$ bound on the difference of $y-y_{c}$ at the expense of additional regularity assumptions was obtained. Let us set

$$
\mathcal{A}=\{x \in \Omega: y(x)=\psi(x)\}, \quad \mathcal{A}_{c}=\left\{x \in \Omega: y_{c}(x)=\psi(x)\right\} .
$$

Assume that $u \in L^{\infty}(\Omega)$. If, moreover, the boundary $\partial \mathcal{A}$ of the active set is a $C^{1,1}$ manifold in $\mathbb{R}^{n-1}$ and for every $c>0$ the boundary $\partial \mathcal{A}_{c}$ of $\mathcal{A}_{c}$ is a Lipschitzian manifold in $\mathbb{R}^{n-1}$, then

$$
\left\|y_{c}-y\right\|_{L^{\infty}(\Omega)} \leq \frac{1}{c}\|u-A \psi\|_{L^{\infty}(\Omega)} .
$$

In the following subsection we shall see that setting $\bar{\lambda}$ large enough yields feasibility of $y_{c}$. This enables to give $L^{\infty}$-convergence results of the following type.

Proposition 3.4. Let $\bar{\lambda} \in L^{\infty}(\Omega)$ be given and suppose that $y_{c} \leq \psi$. Then

$$
\left\|y-y_{c}\right\|_{L^{\infty}} \leq \frac{\|\bar{\lambda}\|_{L^{\infty}}}{c}+\frac{1}{2 c^{2}}
$$

Proof. Let us define the test function

$$
\phi_{k}= \begin{cases}y-y_{c}-k & \text { if } y-y_{c}>k \\ y-y_{c}+k & \text { if } y-y_{c}<-k \\ 0 & \text { otherwise }\end{cases}
$$

where $k \in \mathbb{R}$. Proceeding as in the proof for Lemma 3.2 we get

$$
a\left(y-y_{c}, \phi_{k}\right)+\left\langle\lambda-\max _{c}\left(0, \bar{\lambda}+c\left(y_{c}-\psi\right)\right), \phi_{k}\right\rangle=0 .
$$

We can split $\phi_{k}=\max \left(\phi_{k}, 0\right)+\min \left(\phi_{k}, 0\right)$, since both addends belong to $H_{0}^{1}(\Omega)$. As in the previous proof we obtain $\left\langle\lambda, \max \left(\phi_{k}, 0\right)\right\rangle \geq 0$. By feasibility of $y_{c}$ we find

$$
0 \geq \min \left(\phi_{k}, 0\right)=\min \left(y-y_{c}+k, 0\right) \geq \min (y-\psi+k, 0) \geq \min (y-\psi, 0)=y-\psi .
$$

This implies

On the set $\left\{\phi_{k}>0\right\}$, we find

$$
0 \geq\left\langle\lambda, \min \left(\phi_{k}, 0\right)\right\rangle \geq\langle\lambda, y-\psi\rangle=0
$$

$$
\max _{c}\left(0, \bar{\lambda}+c\left(y_{c}-\psi\right)\right)=0 \text { for } k \geq \frac{\|\bar{\lambda}\|_{L^{\infty}}}{c}+\frac{1}{2 c^{2}},
$$

and hence $\left(\max _{c}\left(0, \bar{\lambda}+c\left(y_{c}-\psi\right)\right), \max \left(\phi_{k}, 0\right)\right)=0$. Since from the definition $\max _{c}\left(0, \bar{\lambda}+c\left(y_{c}-\psi\right)\right) \geq 0$, we have

$$
-\left(\max _{c}\left(0, \bar{\lambda}+c\left(y_{c}-\psi\right)\right), \min \left(\phi_{k}, 0\right)\right) \geq 0
$$

Thus, we obtained $a\left(y-y_{c}, \phi_{k}\right) \leq 0$ for $k \geq \frac{\|\bar{\lambda}\|_{L^{\infty}}}{c}+\frac{1}{2 c^{2}}$. Due to the properties of the bilinear form $a$ and the definition of $\phi_{k}$, we have $a\left(y-y_{c}, \phi_{k}\right) \geq a\left(\phi_{k}, \phi_{k}\right)$, which implies $\phi_{k}=0$.

Remark 3.5. Analogously to Remark 3.3 a slightly tighter estimate for the feasible case was obtained in [13] under additional regularity requirements. In fact, if $\mathcal{A}_{c}$ is a domain with a $C^{1,1}$ boundary, then

$$
\left\|y_{c}-y\right\|_{L^{\infty}(\Omega)} \leq \frac{1}{c}\|\bar{\lambda}\|_{L^{\infty}(\Omega)} .
$$


Up to now, we studied convergence of solutions for fixed right-hand side $u$ in (3.1). Let us now turn to the case, where the right-hand side is a (possibly weakly) convergent sequence. Due to the monotonicity of the $\max _{c}$-function we obtain the following Lipschitz continuity result for the solutions of the regularized equation.

Lemma 3.6. Let $\bar{\lambda} \in L^{\infty}(\Omega)$ be given. Let $u_{1}, u_{2} \in H^{-1}(\Omega)$ be given. Then there exists a constant L $>0$ independent of $c$ such that

$$
\left\|y_{c}\left(u_{1}\right)-y_{c}\left(u_{2}\right)\right\|_{H_{0}^{1}} \leq L\left\|u_{1}-u_{2}\right\|_{H^{-1}} .
$$

Lemma 3.7. Let $\bar{\lambda} \in L^{\infty}(\Omega)$ be given. Let $u_{c} \rightarrow u$ in $L^{2}(\Omega)$. Then $y_{c} \rightarrow y$ in $H_{0}^{1}(\Omega)$ strongly.

Proof. It holds

$$
\left\|y_{c}\left(u_{c}\right)-y(u)\right\|_{H^{1}} \leq\left\|y_{c}\left(u_{c}\right)-y_{c}(u)\right\|_{H^{1}}+\left\|y_{c}(u)-y(u)\right\|_{H^{1}} .
$$

The first addend can be majorized by $L\left\|u_{c}-u\right\|_{H^{-1}}$ due to Lemma 3.6. By compact embeddings this term tends to zero for $c \rightarrow \infty$. The second addend tends to zero according to Lemma 3.1.

The final result of this section addresses convergence of the solutions of the regularized optimal control problem $\left(\mathrm{P}_{c}\right)$ to those of original problem $(\mathrm{P})$.

Proposition 3.8. Let $j: L^{2}(\Omega) \rightarrow \mathbb{R}$ be weakly lower semi-continuous.

For every $c>0$ problem $\left(\mathrm{P}_{c}\right)$ admits a global solution $\left(y_{c}, u_{c}\right)=\left(y\left(u_{c}\right), u_{c}\right) \in H_{0}^{1}(\Omega) \times L^{2}(\Omega)$. For every subsequence of controls $\left\{u_{c_{n}}\right\}$ converging weakly in $L^{2}(\Omega)$ (of which there exists at least one) to some $u^{*}$, the corresponding states $y_{c_{n}}=y\left(u_{c_{n}}\right)$ converge strongly in $H_{0}^{1}(\Omega)$ to $y^{*}=y\left(u^{*}\right)$, and $\left(y^{*}, u^{*}\right)$ is a global solution to $(\mathrm{P})$. Moreover $\lambda_{c_{n}}=\max _{c}\left(0, \bar{\lambda}+c_{n}\left(y_{c_{n}}-\psi\right)\right) \rightarrow \lambda\left(u^{*}\right)$ weakly in $H^{-1}(\Omega)$.

In addition, in the feasible case with $y_{c_{n}} \leq \psi$ for all $n,\left\{\left(p_{c_{n}}, \mu_{c_{n}}\right)\right\}$ converge weakly in $H_{0}^{1}(\Omega)$ and weakly star in $L^{\infty}(\Omega)^{*}$ to $\left(p^{*}, \mu^{*}\right) \in H_{0}^{1}(\Omega) \times L^{\infty}(\Omega)^{*}$ satisfying $(2.3)-(2.7)$.

Proof. Since $j$ is radially unbounded and $g$ is bounded from below, every minimizing sequence $\left\{\left(y_{c}\left(u_{n}\right), u_{n}\right)\right\}$ to $\left(\mathrm{P}_{c}\right)$ has a weakly convergent subsequence, denoted by the same symbol, with weak limit $u_{c} \in L^{2}(\Omega)$. By Lemma 3.6 we find $y_{c}\left(u_{n}\right) \rightarrow y_{c}\left(u_{c}\right)$ strongly in $H_{0}^{1}(\Omega)$. Weak lower semi-continuity of $j$ and continuity of $g: H_{0}^{1}(\Omega) \rightarrow \mathbb{R}$ imply that $\left(y_{c}\left(u_{c}\right), u_{c}\right)$ is a solution to $\left(\mathrm{P}_{c}\right)$.

Next consider a family of solutions $\left\{\left(y_{c}, u_{c}\right)\right\}$ to $\left(\mathrm{P}_{c}\right)$. Let $y_{c}(0)$ denote the solution to the equality constraint in $\left(\mathrm{P}_{c}\right)$ with $u=0$, and note that $\left\{y_{c}(0)\right\}_{c} \geq 1$ is bounded in $H_{0}^{1}(\Omega)$. Hence $\left\{g\left(y_{c}(0)\right)\right\}_{c>1}$ is bounded as well. Then $\left(y_{c}(0), 0\right)$ is a feasible pair for $\left(\mathrm{P}_{c}\right)$ for every $c>0$, and $J\left(y_{c}, u_{c}\right) \leq J\left(y_{c}(0), 0\right)$. Thus $\left\{j\left(u_{c}\right)\right\}_{c \geq 1}$ is bounded and radial unboundedness of $j$ implies that $\left\{u_{c}\right\}_{c>1}$ is bounded in $L^{2}(\Omega)$. Consequently there exists a weakly convergent subsequence $u_{c_{n}}$ in $L^{2}(\Omega)$ with weak limit $u^{*} \in L^{2}(\Omega)$. By Lemma 3.7 the sequence $y_{c_{n}}=y\left(u_{c_{n}}\right) \rightarrow y\left(u^{*}\right)$ strongly in $H_{0}^{1}(\Omega)$. Moreoever $\lambda_{c_{n}}=\max _{c_{n}}\left(0, \bar{\lambda}+c_{n}\left(y_{c_{n}}-\psi\right)\right) \rightarrow \lambda\left(y^{*}\right)$ strongly in $H^{-1}(\Omega)$, where $A y^{*}+\lambda\left(y^{*}\right)=u^{*}$. As above we can now pass to the limit in $\left(\mathrm{P}_{c_{n}}\right)$ as $n \rightarrow \infty$ and obtain that $\left(y^{*}, u^{*}\right)$ is a solution to $(\mathrm{P})$, with associated Lagrange multiplier $\lambda\left(y^{*}\right)$. By Theorem 2.7 there exists a uniquely associated adjoint state $p^{*} \in H_{0}^{1}(\Omega)$ and $\mu^{*} \in H^{-1}(\Omega) \cap L^{\infty}(\Omega)^{*}$ satisfying $(2.3)-(2.7)$. Convergence of $p_{c_{n}} \rightarrow p^{*}$ and $\mu_{c_{n}} \rightarrow \mu^{*}$ weakly in $H_{0}^{1}(\Omega)$ and weakly star in $L^{\infty}(\Omega)^{*}$ was proved in [12] for the feasible case.

Corollary 3.9. For the quadratic cost $(2.16)$ with $\psi \leq y_{d}$ the family $\left\{\left(u_{c}, y\left(u_{c}\right), \lambda\left(u_{c}\right)\right)\right\}$ converges in $L^{2}(\Omega)_{\text {weak }} \times$ $H_{0}^{1}(\Omega) \times H^{-1}(\Omega)_{\text {weak }}$ to $\left(u^{*}, y\left(u^{*}\right), \lambda\left(u^{*}\right)\right)$, as $c \rightarrow \infty$, where $\left(y^{*}, u^{*}\right)$ is the unique solution to $(\mathrm{P})$.

\subsection{Feasibility of solutions for large $\bar{\lambda}$}

In this short subsection we give a sufficient condition so that for large $\bar{\lambda}$ sufficiently large the optimal states $y_{c}$ of $\left(\mathrm{P}_{c}\right)$ are feasible, i.e. $y_{c} \leq \psi$. The principle idea can be found in Theorem 5.1 of [12], but uniformity with respect to $c$ is not clarified there.

Let us first prove an estimate of on the norm of the violation of the constraint $y \leq \psi$. Let us define for solutions $y_{c}$ of the regularized equation

$$
\zeta_{c}:=\max \left(0, y_{c}-\psi\right)
$$

Then we have $\zeta_{c} \geq 0$ and $\zeta_{c} \in H_{0}^{1}(\Omega)$. In addition, we have the following: 
Lemma 3.10. Let $y_{c}$ be a solution of the regularized equation (3.1) for a given right hand side $u \in L^{2}(\Omega)$. Then the violation of $y_{c} \leq \psi$ can be estimated by

$$
\left\|\zeta_{c}\right\|_{H^{1}}^{2}+c\left\|\zeta_{c}\right\|_{L^{2}}^{2} \leq C c^{-1}\|u-A \psi-\bar{\lambda}\|_{L^{2}}^{2}
$$

with a constant $C$ independent of $c, \bar{\lambda}, u, y_{c}$.

Proof. Let us consider the regularized equation

$$
A y_{c}+\max _{c}\left(0, \bar{\lambda}+c\left(y_{c}-\psi\right)\right)=u \text {. }
$$

Testing this equation with $\zeta_{c}=\max \left(0, y_{c}-\psi\right) \geq 0$ and using the fact that $\max _{c}(0, x) \geq \max (0, x)$ we obtain

$$
\left\langle A y_{c}-A \psi, \zeta_{c}\right\rangle+\left(\max \left(0, \bar{\lambda}+c\left(y_{c}-\psi\right)\right), \zeta_{c}\right) \leq\left(u-A \psi, \zeta_{c}\right) .
$$

Since $\max (0, x) \geq x$ and $\left(y_{c}-\psi, \zeta_{c}\right)=\left\|\zeta_{c}\right\|_{L^{2}}^{2}$, it follows

$$
\left(\max \left(0, \bar{\lambda}+c\left(y_{c}-\psi\right)\right), \zeta_{c}\right) \geq\left(\bar{\lambda}, \zeta_{c}\right)+c\left\|\zeta_{c}\right\|_{L^{2}}^{2}
$$

and the claim follows with

$$
\left\langle A \zeta_{c}, \zeta_{c}\right\rangle+c\left\|\zeta_{c}\right\|_{L^{2}}^{2} \leq\left(u-A \psi-\bar{\lambda}, \zeta_{c}\right)
$$

by the assumption on the elliptic operator $A$.

The main result of this section is a consequence of estimate (3.2). If $\bar{\lambda}$ is chosen large enough then the solutions $y_{c}$ are feasible, i.e. $y_{c} \leq \psi$.

Proposition 3.11. Let $\rho \geq 0$ and let $y_{c} \in H_{0}^{1}(\Omega)$ denote the solution to $A y_{c}+\max _{c}\left(0, \bar{\lambda}+c\left(y_{c}-\psi\right)\right)=u$ with $u \in B_{\rho}=\left\{u:\|u\|_{L^{\infty}} \leq \rho\right\}$. If $\bar{\lambda} \geq \max (0, \rho-A \psi)$ then $y_{c}$ is feasible, i.e. $y_{c} \leq \psi$ for each $c>0$.

Proof. Let $u \in B_{\rho}$. Due to the assumption on $\bar{\lambda}$ we have since $\zeta_{c} \geq 0$

$$
\left(u-A \psi-\bar{\lambda}, \zeta_{c}\right) \leq\left(\rho-A \psi-\max (0, \rho-A \psi), \zeta_{c}\right) \leq 0 .
$$

Then equation (3.2) implies $\zeta_{c}=0$ and hence $y_{c} \leq \psi$.

Corollary 3.12. Assume that $\left\|j^{\prime}(u)\right\|_{L^{\infty}(\Omega)} \geq K\left(\|u\|_{L^{\infty}(\Omega)}-1\right)$ for a constant $K$ independent of $u \in L^{\infty}(\Omega)$. Then there exists $\rho>0$ such that the optimal controls $u_{c}$ to $\left(\mathrm{P}_{c}\right)$ satisfy $\left\|u_{c}\right\|_{L^{\infty}(\Omega)} \leq \rho$ for all $c \geq 1$ and hence Proposition 3.11 applies.

Proof. The family of solutions $\left\{u_{c}\right\}_{c \geq 1}$ is bounded in $L^{2}(\Omega)$. Hence $\left\{y_{c}\right\}_{c \geq 1}$ is bounded in $H_{0}^{1}(\Omega)$ and $\left\{g^{\prime}\left(y_{c}\right)\right\}_{c \geq 1}$ is bounded in $L^{2}(\Omega)$. Next consider the adjoint equations

$$
A^{*} p_{c}+c \operatorname{sgn}_{c}\left(\bar{\lambda}+c\left(y_{c}-\psi\right)\right) p_{c}=-g^{\prime}\left(y_{c}\right) .
$$

By the Stampacchia method the family of solutions $\left\{p_{c}\right\}_{c \geq 1}$ is bounded in $L^{\infty}(\Omega)$ independently of $\bar{\lambda}$. The claim now follows from the assumption that $\left\|p_{c}\right\|_{L^{\infty}(\Omega)}=\left\|j^{\prime}\left(u_{c}\right)\right\|_{L^{\infty}(\Omega)} \geq K\left(\left\|u_{c}\right\|_{L^{\infty}(\Omega)}-1\right)$.

Remark 3.13. Let us mention that the choice $j(u)=\frac{\beta}{2}\|u\|_{L^{2}}^{2}, \beta>0$, fulfills the requirements of this corollary. In fact, $j^{\prime}(u)=\beta u$, which shows that the condition is satisfied with $K=\beta$. 


\subsection{Existence of approximating families}

Let $\left(y^{*}, u^{*}\right)$ be a strictly locally optimal pair for $(\mathrm{P})$, that is, there exists $\rho>0$ such that

$$
J\left(y^{*}, u^{*}\right)<J(y, u) \quad \text { for all }(y, u) \text { satisfying (1.3) and } 0<\left\|u-u^{*}\right\|_{L^{2}}<\rho .
$$

We will show that for each strictly locally optimal pair $\left(y^{*}, u^{*}\right)$ there is a family of local solutions $\left(y_{c}, u_{c}\right)$ of $\left(\mathrm{P}_{c}\right)$ that converges strongly to $\left(y^{*}, u^{*}\right)$ in $H_{0}^{1}(\Omega) \times L^{2}(\Omega)$. This means in particular, we show existence of a path of solutions $\left\{y_{c}, u_{c}\right\}_{c>c_{0}}$.

Theorem 3.14. Let $j$ be weakly lower semi-continuous. Moreover, we require for $j$ that

$$
u_{n} \rightarrow u \text { in } L^{2}(\Omega) \text {, and } j\left(u_{n}\right) \rightarrow j(u) \text { imply } u_{n} \rightarrow u \text { in } L^{2}(\Omega) .
$$

Let $\left(y^{*}, u^{*}\right)$ be a strictly locally optimal pair for $(\mathrm{P})$. Then there exists a family of local solutions $\left(y_{c}, u_{c}\right)$ of $\left(\mathrm{P}_{c}\right)$ that converges strongly to $\left(y^{*}, u^{*}\right)$ in $H_{0}^{1}(\Omega) \times L^{2}(\Omega)$.

The pre-requisite (3.4) is fulfilled for instance for the standard choice $j(u)=\frac{\beta}{2}\|u\|_{L^{2}}^{2}, \beta>0$.

Proof. Let $\rho$ be given by (3.3) and take $\rho^{\prime}$ with $0<\rho^{\prime}<\rho$. Consider the auxiliary problem

$$
\left\{\begin{array}{l}
\text { Minimize } J(y, u)=g(y)+j(u) \\
\text { over } u \in L^{2}(\Omega) \text { with }\left\|u-u^{*}\right\|_{L^{2}} \leq \rho^{\prime} \text { and subject to } \\
A y+\max _{c}(0, \bar{\lambda}+c(y-\psi))=u .
\end{array}\right.
$$

Clearly, this optimal control problem is solvable for every $c>0$. Let $u_{c}$ denote a global solution of $\left(\mathrm{P}_{c}^{\rho^{\prime}}\right)$. By construction, the set $\left\{u_{c}\right\}_{c>0}$ is bounded, which yields weak convergence of a subsequence $u_{c_{n}} \rightarrow \tilde{u}$ in $L^{2}(\Omega)$ with $\left\|\tilde{u}-u^{*}\right\|_{L^{2}} \leq \rho^{\prime}$. Due to Lemma 3.7, the corresponding states converge $y_{c_{n}} \rightarrow \tilde{y}$ in $H_{0}^{1}(\Omega)$, where $\tilde{y}$ is a solution of the variational inequality (1.3) for right-hand side $\tilde{u}$. This implies $J\left(y^{*}, u^{*}\right) \leq J(\tilde{y}, \tilde{u})$ by optimality of $\left(y^{*}, u^{*}\right)$.

Denoting by $y_{c}\left(u^{*}\right)$ the solution of the regularized equation to the control $u^{*}$, we have $J\left(y_{c}, u_{c}\right) \leq J\left(y_{c}\left(u^{*}\right), u^{*}\right)$. By Lemma 3.1, we have $y_{c}\left(u^{*}\right) \rightarrow y^{*}$ in $H_{0}^{1}(\Omega)$.

Then we obtain by (3.3) and the optimality and convergence properties above

$$
\begin{aligned}
g\left(y^{*}\right)+j\left(u^{*}\right) & \leq g(\tilde{y})+j(\tilde{u}) \leq \lim g\left(y_{c_{n}}\right)+\liminf j\left(u_{c_{n}}\right) \\
& \leq \lim g\left(y_{c_{n}}\right)+\limsup j\left(u_{c_{n}}\right) \leq \lim g\left(y_{c_{n}}\left(u^{*}\right)\right)+j\left(u^{*}\right) \\
& =g\left(y^{*}\right)+j\left(u^{*}\right) .
\end{aligned}
$$

This implies $J\left(y^{*}, u^{*}\right)=J(\tilde{y}, \tilde{u})$ and hence $\tilde{u}=u^{*}$ by strict local optimality of $\left(y^{*}, u^{*}\right)$. Moreover, it follows that $\lim j\left(u_{c_{n}}\right)=j(\tilde{u})$ which yields $u_{c_{n}} \rightarrow u^{*}$ in $L^{2}(\Omega)$ by (3.4). Since $u^{*}$ is the unique local solution in the $L^{2}$-neighborhood of $u^{*}$ of radius $\rho^{\prime}$, the whole family $u_{c}$ converges to $u^{*}$.

Convergence of $u_{c} \rightarrow u^{*}$ also implies the existence of $c_{0}$ such that $\left\|u_{c}-u^{*}\right\|_{L^{2}}<\rho^{\prime}$ for all $c>c_{0}$. Consequently, if $c>c_{0}$, then $\left(y_{c}, u_{c}\right)$ is locally optimal for $\left(\mathrm{P}_{c}\right)$.

Regarding convergence of adjoint states and multipliers, we obtain:

Corollary 3.15. Let $\left(y_{c}, u_{c}\right)$ be a family of local solutions of $\left(\mathrm{P}_{c}\right)$ converging strongly in $H_{0}^{1}(\Omega) \times L^{2}(\Omega)$ to $\left(y^{*}, u^{*}\right)$. Let $\left(y^{*}, u^{*}\right)$ solve the variational inequality and satisfy together with $\left(\lambda^{*}, p^{*}, \mu^{*}\right)$ the first-order optimality system (2.3)-(2.8) given by Theorem 2.7. Then we have

$$
\lambda_{c} \rightarrow \lambda^{*} \text { and } \mu_{c} \rightarrow \mu^{*} \text { in } H^{-1}(\Omega), \quad p_{c} \rightarrow p^{*} \text { in } H_{0}^{1}(\Omega),
$$

where $\left(\lambda_{c}, p_{c}, \mu_{c}\right)$ are the corresponding multipliers and adjoint state for $\left(\mathrm{P}_{c}\right)$, see (1.7). 
Proof. Due to the strong convergence of $y_{c}, u_{c}$, the strong convergence of $\lambda_{c}$ follow immediately

$$
\lambda_{c}=u_{c}-A y_{c} \rightarrow u^{*}-A y^{*}=\lambda^{*} \text { in } H^{-1}(\Omega) .
$$

Multiplying the second equation in (1.7) by $p_{c}$ gives

$$
\nu_{1}\left\|p_{c}\right\|_{H^{1}}^{2} \leq\left\|g^{\prime}\left(y_{c}\right)\right\|_{L^{2}}\left\|p_{c}\right\|_{L^{2}},
$$

which gives boundedness of $\left\{p_{c}\right\}$ in $H_{0}^{1}(\Omega)$. Hence, we find a subsequence $p_{c_{n}}$ converging weakly in $H_{0}^{1}(\Omega)$ and strongly in $L^{2}(\Omega)$ to $\tilde{p}$. The third equation in (1.7) implies

$$
j^{\prime}\left(u_{c_{n}}\right)=p_{c_{n}} \rightarrow j^{\prime}\left(u^{*}\right)=\tilde{p},
$$

which gives $\tilde{p}=p^{*}$ by optimality condition (2.7). Since the adjoint state $p^{*}$ is uniquely determined by $\left(y^{*}, u^{*}\right)$, the whole family $p_{c}$ converges weakly in $H_{0}^{1}(\Omega)$ to $p^{*}$. Arguing as above, we find for $\mu_{c}$

$$
\mu_{c}=-A^{*} p_{c}-g^{\prime}\left(y_{c}\right) \rightarrow-A^{*} p^{*}-g^{\prime}\left(y^{*}\right)=\mu^{*} \text { in } H^{-1}(\Omega),
$$

which finishes the proof.

\subsection{Sufficient optimality condition for the regularized problems}

The focus of this section lies on a second order sufficient optimality condition. However, to appreciate the condition that will be used, we first derive a second order necessary condition.

We shall frequently use the second derivative of the $\max _{c}$-function, which is given by

$$
\operatorname{sgn}_{c}^{\prime}(x)= \begin{cases}0, & \text { for }|x|>\frac{1}{2 c} \\ c, & \text { for }|x| \leq \frac{1}{2 c}\end{cases}
$$

Theorem 3.16. Let $\left(y_{c}, u_{c}\right)$, with $y_{c}=y\left(u_{c}\right)$, denote a local minimizer for the regularized problem $\left(\mathrm{P}_{c}\right)$ with associated multipliers $\left(\lambda_{c}, p_{c}, \mu_{c}\right)$ and assume that

$$
\operatorname{meas}\left(\left\{\left|\bar{\lambda}+c\left(y_{c}-\psi\right)\right|=\frac{1}{2 c}\right\}\right)=0
$$

Then

$$
g^{\prime \prime}\left(y_{c}\right)(z, z)+j^{\prime \prime}\left(u_{c}\right)(h, h)+\left(c^{2} \operatorname{sgn}_{c}^{\prime}\left(\bar{\lambda}+c\left(y_{c}-\psi\right)\right) p_{c} z, z\right) \geq 0,
$$

for all $(z, h) \in H_{0}^{1}(\Omega) \times L^{2}(\Omega)$ satisfying

$$
A z+c \operatorname{sgn}_{c}\left(\bar{\lambda}+c\left(y_{c}-\psi\right)\right) z=h .
$$

The variable $z$ is the directional derivative of $u \rightarrow y(u)$ in direction $h$. For $\bar{\lambda}=0$ condition (3.8) excludes the case where $y_{c}$ is at constant distance to the obstacle on the a set of nontrivial measure. This condition is not required if more regular approximations to the max function are utilized.

Proof of Theorem 3.16. Let $h \in L^{2}(\Omega)$ be arbitrary and set

$$
u_{k}=u_{c}+\rho_{k} h, \text { with } \lim _{k \rightarrow \infty} \rho_{k}=0, \text { and } y_{k}=y_{c}\left(u_{k}\right) .
$$


Due to monotonicity of $y \rightarrow \max _{c}(\bar{\lambda}+c(y-\psi))$ it can be argued that the sequence $z_{k}:=\frac{1}{\rho_{k}}\left(y_{k}-y_{c}\right)$, is bounded in $H_{0}^{1}(\Omega)$. Since $\max _{c}$ is continuously differentiable with Lipschitz continuous first derivative, and the mapping $u \mapsto y_{c}(u)$ is Lipschitz continuous from $H^{-1}(\Omega)$ to $H_{0}^{1}(\Omega)$, strong convergence

$$
z_{k} \rightarrow z \text { in } H_{0}^{1}(\Omega)
$$

can be verified as for semilinear elliptic equations, see e.g. [7]. Moreover, it follows that $(z, h)$ solves the linearized equation

$$
A z+c \operatorname{sgn}_{c}\left(\bar{\lambda}+c\left(y_{c}-\psi\right)\right) z=h .
$$

Since the solution to this equation is unique this argument also shows the directional differentiability of $u \rightarrow y(u)$ from $L^{2}(\Omega)$ to $H_{0}^{1}(\Omega)$.

Let us investigate the difference of the values of the objective functional $J$. Since $y_{k}$ and $y_{c}$ are solutions of the regularized state equation, we obtain

$$
\begin{aligned}
J\left(y_{k}, u_{k}\right)-J\left(y_{c}, u_{c}\right)= & J\left(y_{k}, u_{k}\right)-J\left(y_{c}, u_{c}\right)+\left\langle A\left(y_{k}-y_{c}\right)\right. \\
& \left.+\max _{c}\left(0, \bar{\lambda}+c\left(y_{k}-\psi\right)\right)-\max _{c}\left(0, \bar{\lambda}+c\left(y_{c}-\psi\right)\right)-\left(u_{k}-u_{c}\right), p_{c}\right\rangle .
\end{aligned}
$$

Due to condition (3.7), we can expand

$$
\begin{aligned}
\max _{c}\left(0, \bar{\lambda}+c\left(y_{k}-\psi\right)\right) & -\max _{c}\left(0, \bar{\lambda}+c\left(y_{c}-\psi\right)\right) \\
= & c \operatorname{sgn}_{c}\left(\bar{\lambda}+c\left(y_{c}-\psi\right)\right)\left(y_{k}-y_{c}\right)+\frac{1}{2} c^{2} \operatorname{sgn}_{c}^{\prime}\left(\bar{\lambda}+c\left(y_{c}-\psi\right)\right)\left(y_{k}-y_{c}\right)^{2} \\
& +\int_{0}^{1} \int_{0}^{s}\left\{c^{2} \operatorname{sgn}_{c}^{\prime}\left(\bar{\lambda}+c\left(y_{c}+t\left(y_{k}-y_{c}\right)-\psi\right)\right)-c^{2} \operatorname{sgn}_{c}^{\prime}\left(\bar{\lambda}+c\left(y_{c}-\psi\right)\right)\right\}\left(y_{k}-y_{c}\right)^{2} \mathrm{~d} t \mathrm{~d} s .
\end{aligned}
$$

Applying Taylor expansion to all non-linear terms in (3.11) and using the optimality system (1.7), we find

$$
\begin{aligned}
0 \leq & J\left(y_{k}, u_{k}\right)-J\left(y_{c}, u_{c}\right)=J\left(y_{k}, u_{k}\right)-J\left(y_{c}, u_{c}\right) \\
& +\left\langle A\left(y_{k}-y_{c}\right)+\max _{c}\left(0, \bar{\lambda}+c\left(y_{k}-\psi\right)\right)-\max _{c}\left(0, \bar{\lambda}+c\left(y_{c}-\psi\right)\right)-\left(u_{k}-u_{c}\right), p_{c}\right\rangle \\
= & g^{\prime}\left(y_{c}\right)\left(y_{k}-y_{c}\right)+\frac{1}{2} g^{\prime \prime}\left(\tilde{y}_{k}\right)\left(y_{k}-y_{c}\right)^{2} \\
& +\left(j^{\prime}\left(u_{c}\right)-p_{c}, u_{k}-u_{c}\right)+\frac{1}{2} j^{\prime \prime}\left(\tilde{u}_{k}\right)\left(u_{k}-u_{c}\right)^{2} \\
& +\left\langle A^{*} p_{c}, y_{k}-y_{c}\right\rangle+\left(c \operatorname{sgn}_{c}\left(\bar{\lambda}+c\left(y_{c}-\psi\right)\right)\left(y_{k}-y_{c}\right), p_{c}\right) \\
& +\frac{1}{2}\left(c^{2} \operatorname{sgn}_{c}^{\prime}\left(\bar{\lambda}+c\left(y_{c}-\psi\right)\right)\left(y_{k}-y_{c}\right)^{2}, p_{c}\right) \\
& +\int_{0}^{1} \int_{0}^{s}\left(\left\{c^{2} \operatorname{sgn}_{c}^{\prime}\left(\bar{\lambda}+c\left(y_{c}+t\left(y_{k}-y_{c}\right)-\psi\right)\right)-c^{2} \operatorname{sgn}_{c}^{\prime}\left(\bar{\lambda}+c\left(y_{c}-\psi\right)\right)\right\}\left(y_{k}-y_{c}\right)^{2}, p_{c}\right) \mathrm{d} t \mathrm{~d} s \\
= & \frac{1}{2}\left(g^{\prime \prime}\left(\tilde{y}_{k}\right)\left(y_{k}-y_{c}\right)^{2}+j^{\prime \prime}\left(\tilde{u}_{k}\right)\left(u_{k}-u_{c}\right)^{2}+\left(c^{2} \operatorname{sgn}_{c}^{\prime}\left(\bar{\lambda}+c\left(y_{c}-\psi\right)\right)\left(y_{k}-y_{c}\right)^{2}, p_{c}\right)\right) \\
& +\int_{0}^{1} \int_{0}^{s}\left(\left\{c^{2} \operatorname{sgn}_{c}^{\prime}\left(\bar{\lambda}+c\left(y_{c}+t\left(y_{k}-y_{c}\right)-\psi\right)\right)-c^{2} \operatorname{sgn}_{c}^{\prime}\left(\bar{\lambda}+c\left(y_{c}-\psi\right)\right)\right\}\left(y_{k}-y_{c}\right)^{2}, p_{c}\right) \mathrm{d} t \mathrm{~d} s
\end{aligned}
$$


where $\tilde{u}_{k}$ and $\tilde{y}_{k}$ lie between $u_{k}$ and $u_{c}$, and $y_{k}$ and $y_{c}$, respectively. Dividing by $\rho_{k}^{2}$ gives

$$
\begin{aligned}
0 \leq \frac{1}{2}\left(g^{\prime \prime}\left(\tilde{y}_{k}\right)\left(z_{k}\right)^{2}+j^{\prime \prime}\left(\tilde{u}_{k}\right)(h)^{2}+\left(c^{2} \operatorname{sgn}_{c}^{\prime}\left(\bar{\lambda}+c\left(y_{c}-\psi\right)\right) z_{k}^{2}, p_{c}\right)\right) \\
\quad+\int_{0}^{1} \int_{0}^{s}\left(\left\{c^{2} \operatorname{sgn}_{c}^{\prime}\left(\bar{\lambda}+c\left(y_{c}+t\left(y_{k}-y_{c}\right)-\psi\right)\right)-c^{2} \operatorname{sgn}_{c}^{\prime}\left(\bar{\lambda}+c\left(y_{c}-\psi\right)\right)\right\} z_{k}^{2}, p_{c}\right) \mathrm{d} t \mathrm{~d} s .
\end{aligned}
$$

Below we shall prove that the term containing the double integral tends to 0 for $k \rightarrow \infty$. The claim then follows from (3.12).

Let us investigate

$$
\begin{aligned}
\int_{\Omega}\left\{c^{2} \operatorname{sgn}_{c}^{\prime}\left(\bar{\lambda}+c\left(y_{c}+t\left(y_{k}-y_{c}\right)-\psi\right)\right)-c^{2} \operatorname{sgn}_{c}^{\prime}\right. & \left.\left(\bar{\lambda}+c\left(y_{c}-\psi\right)\right)\right\} z_{k}^{2} p_{c} \\
& =\int_{\left\{\left|\bar{\lambda}+c\left(y_{c}+t\left(y_{k}-y_{c}\right)-\psi\right)\right| \leq \frac{1}{2 c}\right\}} c^{3} z_{k}^{2} p_{c}-\int_{\left\{\left|\bar{\lambda}+c\left(y_{c}-\psi\right)\right| \leq \frac{1}{2 c}\right\}} c^{3} z_{k}^{2} p_{c} .
\end{aligned}
$$

We will prove that this term tends to zero for $k \rightarrow \infty$ uniformly with respect to the parameter $t$. Let us denote $f_{t, k}:=\bar{\lambda}+c\left(y_{c}+t\left(y_{k}-y_{c}\right)-\psi\right), t \in(0,1), f:=\bar{\lambda}+c\left(y_{c}-\psi\right)$. Then we have $\left\|f_{t, k}-f\right\|_{L^{2}} \leq c\left\|y_{k}-y_{c}\right\|_{L^{2}} \rightarrow 0$ for $k \rightarrow \infty$ uniformly with respect to $t$.

Let us prove that the measure of the symmetric difference $N_{t, k}:=\left\{\left|f_{t, k}\right| \leq \frac{1}{2 c}\right\}$ and $N:=\left\{|f| \leq \frac{1}{2 c}\right\}$, denoted by $N_{t, k} \Delta N$, tends to zero uniformly in $t$ for $k \rightarrow \infty$. Consider first

$$
N_{t, k} \backslash N=N_{t, k} \cap\left(\left\{\frac{1}{2 c}<|f|<\frac{1}{2 c}+\delta_{k}\right\} \cup\left\{|f| \geq \frac{1}{2 c}+\delta_{k}\right\}\right)
$$

with $\delta_{k}=\left\|y_{c}-y_{k}\right\|_{L^{2}}^{1 / 2}$. On $N_{t, k} \cap\left\{|f| \geq \frac{1}{2 c}+\delta_{k}\right\}$ we find $\left|f_{t, k}(x)-f(x)\right| \geq \delta_{k}$, and hence by the Tchebychev inequality

$$
\begin{aligned}
\operatorname{meas}\left(N_{t, k} \cap\left\{|f| \geq \frac{1}{2 c}+\delta_{k}\right\}\right) & \leq \operatorname{meas}\left(\left\{\left|f_{t, k}-f\right| \geq \delta_{k}\right\}\right) \\
& \leq \frac{1}{\delta_{k}^{2}}\left\|f-f_{t, k}\right\|_{L^{2}}^{2} \leq c^{2}\left\|y_{c}-y_{k}\right\|_{L^{2}}
\end{aligned}
$$

Moreover, due to its construction meas $\left(\left\{\frac{1}{2 c}<|f|<\frac{1}{2 c}+\delta_{k}\right\}\right) \rightarrow 0$, which implies meas $\left(N_{t, k} \backslash N\right) \rightarrow 0$ for $k \rightarrow \infty$ uniform in $t$.

Let us choose $k_{0}$ such that $\delta_{k}<\frac{1}{2 c}$ for all $k>k_{0}$. Then we write

$$
N \backslash N_{t, k}=\left(\left\{|f|<\frac{1}{2 c}-\delta_{k}\right\} \cup\left\{\frac{1}{2 c}-\delta_{k} \leq|f| \leq \frac{1}{2 c}\right\}\right) \cap\left\{\left|f_{t, k}\right|>\frac{1}{2 c}\right\} .
$$

Here, we find meas $\left(\left\{|f|<\frac{1}{2 c}-\delta_{k}\right\} \cap\left\{\left|f_{t, k}\right|>\frac{1}{2 c}\right\}\right) \rightarrow 0$ with analogous arguments as above. Moreover, meas $\left(\left\{\frac{1}{2 c}-\delta_{k} \leq|f| \leq \frac{1}{2 c}\right\}\right) \rightarrow 0$ due to (3.7). Hence, we have shown that the convergence meas $\left(N_{t, k} \Delta N\right) \rightarrow 0$ for $k \rightarrow \infty$ is uniform with respect to $t$. This allows to prove that the remainder term vanishes for $k \rightarrow \infty$ :

$$
\begin{aligned}
& \mid \int_{0}^{1} \int_{0}^{s}\left(\left\{c^{2} \operatorname{sgn}_{c}^{\prime}\left(\bar{\lambda}+c\left(y_{c}+t\left(y_{k}-y_{c}\right)-\psi\right)\right)\right.\right.\left.\left.-c^{2} \operatorname{sgn}_{c}^{\prime}\left(\bar{\lambda}+c\left(y_{c}-\psi\right)\right)\right\} z_{k}^{2}, p_{c}\right) \mathrm{d} t \mathrm{~d} s \mid \\
& \leq \int_{0}^{1} \int_{0}^{s} c^{3} \operatorname{meas}\left(N_{t, k} \Delta N\right)^{1 / 2}\left\|z_{k}\right\|_{L^{4}}^{2}\left\|p_{c}\right\|_{L^{\infty}} \mathrm{d} t \mathrm{~d} s \rightarrow 0 \text { for } k \rightarrow \infty .
\end{aligned}
$$


We are now prepared to address second order sufficient optimality for $\left(\mathrm{P}_{c}\right)$ under the assumption:

Assumption 2. There exist $\beta>0, C_{0}>0$ such that for all $c>C_{0}$

$$
g^{\prime \prime}\left(y_{c}\right)(z, z)+j^{\prime \prime}\left(u_{c}\right)(h, h)+\left(c^{2} \operatorname{sgn}_{c}^{\prime}\left(\bar{\lambda}+c\left(y_{c}-\psi\right)\right) p_{c} z, z\right) \geq \beta\|h\|_{L^{2}}^{2}
$$

for all $(z, h) \in H_{0}^{1}(\Omega) \times L^{2}(\Omega)$ satisfying

$$
A z+c \operatorname{sgn}_{c}\left(\bar{\lambda}+c\left(y_{c}-\psi\right)\right) z=h .
$$

Thus the gap between the necessary and sufficient conditions consists in the uniform lower bound, see Assumption 2 and (3.8). It is therefore consistent with what can be expected from the literature. Sufficient conditions that imply $p_{c} \geq 0$ were already discussed in Remark 2.13 (iii). In particular for the quadratic function (2.16) with $\psi \leq y_{d}$ we have in the feasible case that $p_{c} \geq 0$ for all $c$ sufficiently large, and hence Assumption 2 is satisfied.

Theorem 3.17. Suppose that Assumption 2 holds at a solution $\left(y_{c}, u_{c}, \lambda_{c}, p_{c}, \mu_{c}\right)$ of the first-order system (1.7) and suppose that (3.7) holds. Then for all $c>C_{0}$ there exist positive constants $\beta_{c}, \rho_{c}$ such that

$$
J\left(y_{c}(u), u\right) \geq J\left(y_{c}, u_{c}\right)+\beta_{c}\left\|u-u_{c}\right\|_{L^{2}}^{2}
$$

for all $u \in L^{2}(\Omega)$ with $\left\|u-u_{c}\right\|_{L^{2}}<\rho_{c}$.

Proof. Let us assume that the quadratic growth condition does not hold for some $c>C_{0}$. Then there exist sequences $u_{k}, u_{k} \rightarrow u_{c}$ in $L^{2}(\Omega), y_{k}=y_{c}\left(u_{k}\right)$, such that

$$
J\left(y_{c}, u_{c}\right)+\frac{1}{k}\left\|u_{k}-u_{c}\right\|_{L^{2}}^{2}>J\left(y_{k}, u_{k}\right)
$$

Introducing the quantities $\rho_{k}=\left\|u_{k}-u_{c}\right\|_{L^{2}}$ and $h_{k}=\frac{1}{\rho_{k}}\left(u_{k}-u_{c}\right)$, we get from the above inequality that

$$
J\left(y_{c}, u_{c}\right)+\frac{\rho_{k}^{2}}{k}>J\left(y_{k}, u_{k}\right) .
$$

The sequence $h_{k}$ is bounded in $L^{2}(\Omega)$ thus by compact embedding of $L^{2}(\Omega)$ into $H^{-1}(\Omega)$ and after extracting a subsequence we have $h_{k} \rightarrow h$ in $L^{2}(\Omega)$ and $h_{k} \rightarrow h$ in $H^{-1}(\Omega)$. As in the proof of the previous theorem we argue that the sequence $z_{k}:=\frac{1}{\rho_{k}}\left(y_{k}-y_{c}\right)$, contain a subsequence such that $z_{k} \rightarrow z$ in $H_{0}^{1}(\Omega)$ where

$$
A z+c \operatorname{sgn}_{c}\left(\bar{\lambda}+c\left(y_{c}-\psi\right)\right) z=h .
$$

Proceeding as in the proof of Theorem 3.16 we arrive at

$$
\begin{aligned}
J\left(y_{k}, u_{k}\right)-J\left(y_{c}, u_{c}\right)= & \frac{1}{2}\left(g^{\prime \prime}\left(\tilde{y}_{k}\right)\left(y_{k}-y_{c}\right)^{2}+j^{\prime \prime}\left(\tilde{u}_{k}\right)\left(u_{k}-u_{c}\right)^{2}\right. \\
& \left.+\left(c^{2} \operatorname{sgn}_{c}^{\prime}\left(\bar{\lambda}+c\left(y_{c}-\psi\right)\right)\left(y_{k}-y_{c}\right)^{2}, p_{c}\right)\right) \\
& +\int_{0}^{1} \int_{0}^{s}\left(\left\{c^{2} \operatorname{sgn}_{c}^{\prime}\left(\bar{\lambda}+c\left(y_{c}+t\left(y_{k}-y_{c}\right)-\psi\right)\right)\right.\right. \\
& \left.\left.-c^{2} \operatorname{sgn}_{c}^{\prime}\left(\bar{\lambda}+c\left(y_{c}-\psi\right)\right)\right\}\left(y_{k}-y_{c}\right)^{2} p_{c}\right) \mathrm{d} t \mathrm{~d} s<\frac{1}{k} .
\end{aligned}
$$

Taking the limit as $k \rightarrow \infty$ we obtain

$$
\frac{1}{2} g^{\prime \prime}\left(y_{c}\right) z^{2}+j^{\prime \prime}\left(u_{c}\right) h^{2}+c^{2} \operatorname{sgn}_{c}^{\prime}\left(\bar{\lambda}+c\left(y_{c}-\psi\right)\right) z^{2} p_{c} \leq 0,
$$

with $z$ satisfying (3.14). This contradicts Assumption 2. 
Remark 3.18. We point out that (3.13) is equivalent to

$$
g^{\prime \prime}\left(y_{c}\right)(z, z)+j^{\prime \prime}\left(u_{c}\right)(h, h)-\left(B^{-1} g^{\prime}\left(y_{c}\right), c^{2} \operatorname{sgn}_{c}^{\prime}\left(\bar{\lambda}+c\left(y_{c}-\psi\right)\right) z^{2}\right) \geq \beta\|h\|_{L^{2}}^{2},
$$

where the operator $B=A^{*}+c \operatorname{sgn}_{c}\left(\bar{\lambda}+c\left(y_{c}-\psi\right)\right)$ satisfies the weak maximum principle. Therefore the last additive term on the left hand side of (3.15) is nonpositive if $g^{\prime}\left(y_{c}\right) \leq 0$, and Assumption 2 holds, for example, if $J$ is of the form (2.16).

In the final result of this section we address the question to which extent Assumption 2 is implied by Assumption 1. It will be necessary to enlarge the test space for in (ii) of Assumption 1 and, in reference to (iii) of Assumption 1, we require that the nonnegativity of $p_{c}$ holds in a small neighborhood of the incidence set.

\section{Assumption 1'.}

(i) There is $\gamma>0$ such that

$$
j^{\prime \prime}\left(u^{*}\right)(h, h) \geq \gamma\|h\|_{L^{2}}^{2} \quad \text { for all } h \in L^{2}(\Omega) .
$$

(ii')

$$
g^{\prime \prime}\left(y^{*}\right)(z, z)+j^{\prime \prime}\left(u^{*}\right)(h, h)>0
$$

holds for all $(z, h, \eta) \in H_{0}^{1}(\Omega) \times L^{2}(\Omega) \times H^{-1}(\Omega, h \neq 0$, satisfying

$$
j^{\prime}\left(u^{*}\right) h+g^{\prime}\left(y^{*}\right) z=0
$$

and

$$
\begin{gathered}
A z+\eta=h \\
\left\langle\eta, y^{*}-\psi\right\rangle=0,\langle\eta, z\rangle \geq 0, \lambda^{*} z=0 \text { a.e. on } \Omega .
\end{gathered}
$$

(iii')

$$
p_{c} \geq 0 \text { on }\left\{\left|\bar{\lambda}+c\left(y_{c}-\psi\right)\right|<1 / 2 c\right\} \text { for all c sufficiently large. }
$$

The test space in Assumption 1' (ii') is larger than in Assumption 1 (ii) since (3.18) allows more test functions than the system in Lemma 2.4 that characterizes the directional derivative $z=y^{\prime}\left(u^{*} ; h\right)$, which is used in Assumption 1. Assumption 1' (iii') implies $\mu_{c} \geq 0$, hence a sign condition on $\mu_{c}$ as in Assumption 1 (iv) is not necessary.

Theorem 3.19. Let $\left\{\left(y_{c}, u_{c}\right)\right\}_{c>0}$ denote a sequence of local solutions to $\left(\mathrm{P}_{c}\right)$ converging to a local solution $\left(y^{*}, u^{*}\right)$ of $(\mathrm{P})$ and let $\bar{\lambda} \geq \Lambda$ be chosen according to Proposition 3.11. Then Assumption 1' implies Assumption 2 for all c sufficiently large.

Proof. Let us suppose that the claim does not hold. Then there exists a sequence $\left\{c_{n}\right\}$ with $\lim _{n \rightarrow \infty} c_{n}=\infty$ and $\left\{\left(z_{c_{n}}, h_{c_{n}}\right)\right\}$ such that

$$
g^{\prime \prime}\left(y_{c_{n}}\right)\left(z_{c_{n}}, z_{c_{n}}\right)+j^{\prime \prime}\left(u_{c_{n}}\right)\left(h_{c_{n}}, h_{c_{n}}\right)+\left(c_{n}^{2} \operatorname{sgn}_{c_{n}}^{\prime}\left(\bar{\lambda}+c_{n}\left(y_{c_{n}}-\psi\right)\right) p_{c_{n}} z_{c_{n}}, z_{c_{n}}\right) \leq \frac{1}{n}\left\|h_{c_{n}}\right\|_{L^{2}}^{2}
$$

with

$$
A z_{c_{n}}+c_{n} \operatorname{sgn}_{c_{n}}\left(\bar{\lambda}+c_{n}\left(y_{c_{n}}-\psi\right)\right) z_{c_{n}}=h_{c_{n}} .
$$

W.l.o.g. we can choose $h_{c_{n}}$ with $\left\|h_{c_{n}}\right\|_{L^{2}}=1$. Then the sequence $z_{c_{n}}$ is bounded in $H_{0}^{1}(\Omega)$. Hence after extracting a subsequence if necessary, we find

$$
h_{c_{n}} \rightarrow h \text { in } L^{2}(\Omega) \text { and } z_{c_{n}} \rightarrow z \text { in } H_{0}^{1}(\Omega) .
$$


Due to the compact embedding $L^{2}(\Omega) \hookrightarrow H^{-1}(\Omega)$ we have $h_{c_{n}} \rightarrow h$ in $H^{-1}(\Omega)$. Passing to the limit in equation (3.20) gives $A z+\eta=h$ together with the convergence $\eta_{c_{n}}:=c_{n} \operatorname{sgn}_{c_{n}}\left(\bar{\lambda}+c_{n}\left(y_{c_{n}}-\psi\right)\right) z_{c_{n}} \rightarrow \eta$ in $H^{-1}(\Omega)$.

Arguing as in [12], pp. 356-357, which uses feasibility $y_{c_{n}} \leq \psi$, we can prove $\left\langle\eta, y^{*}-\psi\right\rangle=0$ and $z \lambda^{*}=0$ a.e. on $\Omega$. Additionally, we obtain $\langle\eta, z\rangle \geq 0$ as follows:

$$
\begin{aligned}
\langle\eta, z\rangle & =\left\langle\eta_{c}, z_{c}\right\rangle+\left\langle\eta-\eta_{c}, z_{c}\right\rangle+\left\langle\eta, z-z_{c}\right\rangle \\
& \geq\left\langle\eta, z-z_{c}\right\rangle+\left\langle\eta-\eta_{c}, z_{c}-z\right\rangle+\left\langle\eta-\eta_{c}, z\right\rangle \\
& =\left\langle\eta, z-z_{c}\right\rangle+\left\langle\eta-\eta_{c}, z\right\rangle+\left\langle h-h_{c}-\left(A z-A z_{c}\right), z_{c}-z\right\rangle \\
& =\left\langle\eta, z-z_{c}\right\rangle+\left\langle\eta-\eta_{c}, z\right\rangle+\left\langle h-h_{c}, z_{c}-z\right\rangle+\left\langle A\left(z-z_{c}\right), z-z_{c}\right\rangle \\
& \geq\left\langle\eta, z-z_{c}\right\rangle+\left\langle\eta-\eta_{c}, z\right\rangle+\left\langle h-h_{c}, z_{c}-z\right\rangle \rightarrow 0,
\end{aligned}
$$

where we used $\left\langle\eta_{c}, z_{c}\right\rangle=\left(c_{n} \operatorname{sgn}_{c_{n}}\left(\bar{\lambda}+c_{n}\left(y_{c_{n}}-\psi\right)\right) z_{c_{n}}, z_{c_{n}}\right) \geq 0$, the defining equations for $\eta_{c}$ and $\eta$, and the coercivity of $A$. These facts establish (3.18).

We have using $h_{c_{n}} \rightarrow h$ in $H^{-1}(\Omega), z_{c_{n}} \rightarrow z, y_{c_{n}} \rightarrow y^{*}$, and $p_{c_{n}} \rightarrow p^{*}$ in $H_{0}^{1}(\Omega)$ and equations (1.7), (3.9)

$$
\begin{aligned}
0=\left(\eta_{c_{n}}, p_{c_{n}}\right)-\left(c_{n} \operatorname{sgn}_{c_{n}}(\bar{\lambda}\right. & \left.\left.+c_{n}\left(y_{c_{n}}-\psi\right)\right) p_{c_{n}}, z_{c_{n}}\right) \\
& =\left\langle h_{c_{n}}, p_{c_{n}}\right\rangle-a\left(z_{c_{n}}, p_{c_{n}}\right)+a\left(z_{c_{n}}, p_{c_{n}}\right)+\left(g^{\prime}\left(y_{c_{n}}\right), z_{c_{n}}\right)=\left\langle h_{c_{n}}, p_{c_{n}}\right\rangle+\left(g^{\prime}\left(y_{c_{n}}\right), z_{c_{n}}\right) \\
& \rightarrow\left\langle h, p^{*}\right\rangle+\left(g^{\prime}\left(y^{*}\right), z\right)=\left\langle h, p^{*}\right\rangle-a\left(z, p^{*}\right)+a\left(z, p^{*}\right)+\left(g^{\prime}\left(y^{*}\right), z\right)=\left\langle\eta, p^{*}\right\rangle-\left\langle\mu^{*}, z\right\rangle,
\end{aligned}
$$

which yields

$$
\left\langle\eta, p^{*}\right\rangle-\left\langle\mu^{*}, z\right\rangle=0
$$

By (2.24), we obtain $j^{\prime}\left(u^{*}\right) h+g^{\prime}\left(y^{*}\right) z=0$, which shows that $(h, z)$ are feasible as test functions in Assumption 1' (ii').

By Assumption 1' (iii'), we have $\left(c_{n}^{2} \operatorname{sgn}_{c_{n}}^{\prime}\left(\bar{\lambda}+c_{n}\left(y_{c_{n}}-\psi\right)\right) p_{c_{n}} z_{c_{n}}, z_{c_{n}}\right) \geq 0$ for sufficiently large $c_{n}>C_{1}$. Hence by (3.19)

$$
g^{\prime \prime}\left(y_{c_{n}}\right)\left(z_{c_{n}}, z_{c_{n}}\right)+j^{\prime \prime}\left(u_{c_{n}}\right)\left(h_{c_{n}}, h_{c_{n}}\right) \leq \frac{1}{n} \quad \text { for all } c_{n}>C_{1} .
$$

We can write

$$
j^{\prime \prime}\left(u_{c_{n}}\right)\left(h_{c_{n}}, h_{c_{n}}\right)=\left(j^{\prime \prime}\left(u_{c_{n}}\right)\left(h_{c_{n}}, h_{c_{n}}\right)-j^{\prime \prime}\left(u^{*}\right)\left(h_{c_{n}}, h_{c_{n}}\right)\right)+j^{\prime \prime}\left(u^{*}\right)\left(h_{c_{n}}, h_{c_{n}}\right),
$$

which gives due to $j^{\prime \prime}\left(u_{c_{n}}\right) \rightarrow j^{\prime \prime}\left(u^{*}\right)$ and Assumption 1' (ii')

$$
\liminf j^{\prime \prime}\left(u_{c_{n}}\right)\left(h_{c_{n}}, h_{c_{n}}\right)=\liminf j^{\prime \prime}\left(u^{*}\right)\left(h_{c_{n}}, h_{c_{n}}\right) \geq j^{\prime \prime}\left(u^{*}\right)(h, h) .
$$

Passing to the limit in (3.21) gives then

$$
g^{\prime \prime}\left(y^{*}\right)(z, z)+j^{\prime \prime}\left(u^{*}\right)(h, h) \leq 0 .
$$

Hence $h=0$ by Assumption 1' (ii'). System (3.18) implies the estimate $\|z\|_{H^{1}} \leq C\|h\|_{H^{-1}}$, which yields $z=0$. Due to Assumption 1' (i) there is $C_{2} \geq C_{1}$ such that

$$
j^{\prime \prime}\left(u_{c_{n}}\right)\left(h_{c_{n}}, h_{c_{n}}\right) \geq \frac{\gamma}{2}\left\|h_{c_{n}}\right\|_{L^{2}}^{2}
$$


for all $c_{n}>C_{2}$. Inequality (3.21) can then be written as

$$
g^{\prime \prime}\left(y_{c_{n}}\right)\left(z_{c_{n}}, z_{c_{n}}\right) \leq \frac{1}{n}-\frac{\gamma}{2} \quad \text { for all } c_{n}>C_{2},
$$

which finally yields the contradiction, since the left-hand side tends to zero for $c_{n} \rightarrow \infty$.

Remark 3.20. If one omits the assumption on $\bar{\lambda}$, one cannot expect feasibility of the regularized solutions $y_{c}$, and consequentely the relations $\left\langle\eta, y^{*}-\psi\right\rangle=0$ and $\lambda^{*} z=0$ cannot be expected to hold. In this case the conclusion of Theorem 3.19 remains correct, if in Assumption 1' (ii') the relations $\left\langle\eta, y^{*}-\psi\right\rangle=0$ and $\lambda^{*} z=0$ are omitted. Note also that the set on which $p_{c}$ is supposed to be nonnegative according to Assumption 1' (iii') is precisely the set on which $\operatorname{sgn}_{c}^{\prime}\left(\bar{\lambda}+c\left(y_{c}-\psi\right)\right)$ does not vanish.

Let us conclude this section with a result about the sets that appear in Assumption 1' (iii').

Lemma 3.21. For every sequence $c_{n} \rightarrow \infty$ the relation

$$
\bigcap_{c_{n}}\left\{\left|\bar{\lambda}+c_{n}\left(y_{c_{n}}-\psi\right)\right|<1 / 2 c_{n}\right\} \subset B,
$$

holds, where $B$ is the bi-active set.

Proof. Let us denote $N_{n}:=\left\{\left|\bar{\lambda}+c_{n}\left(y_{c_{n}}-\psi\right)\right|<1 / 2 c_{n}\right\}$ and $N:=\bigcap_{c_{n}} N_{n}$. Then we have on $N_{n}$

$$
-\frac{1}{2 c_{n}} \leq \bar{\lambda}+c_{n}\left(y_{c_{n}}-\psi\right) \leq \frac{1}{2 c_{n}}
$$

which implies

$$
-\frac{1}{2 c_{n}^{2}}-\bar{\lambda} \frac{1}{c_{n}} \leq y_{c_{n}}-\psi \leq \frac{1}{2 c_{n}^{2}}-\bar{\lambda} \frac{1}{c_{n}} .
$$

Since $y_{c_{n}} \rightarrow y^{*}$ in $L^{p}(\Omega)$, we obtain $y^{*}=\psi$ on $N$ and $y_{c_{n}} \rightarrow y^{*}$ in $L^{\infty}(N)$. In addition, we have

$$
0 \leq \lambda_{c_{n}} \leq \frac{c_{n}}{2}\left(\bar{\lambda}+c_{n}\left(y_{c_{n}}-\psi\right)+\frac{1}{2 c_{n}}\right)^{2} \leq \frac{1}{2 c_{n}}
$$

on $N_{n}$, which proves $\lambda_{c_{n}} \rightarrow 0=\lambda^{*}$ in $L^{\infty}(N)$. Hence, $N \subset B$ holds.

\section{Semi-Smooth NeWton method: Wellposedness And Convergence}

The direct use of Newton-type methods is impeded by the non-smoothness of the constraints appearing in the original problem $(\mathrm{P})$. Even the optimality system $(1.7)$ of the regularized problem $\left(\mathrm{P}_{c}\right)$ contains the nondifferentiable $\operatorname{sgn}_{c}$ term, which is not $C^{1}$, so that Newton's method is not directly applicable. We therefore investigate the use of a semi-smooth Newton method. In view of recent results on semi-smooth methods for non-differentiable problems in function spaces the proof of the semi-smooth part is quite straightforward, see e.g. $[9,13]$. The stability part, however, is more delicate.

The main assumption, under which we obtain well-posed and superlinear convergence of the semi-smooth Newton method, is the second order sufficient optimality condition for the regularized problem that was developed in Section 3.4, specifically (3.13). Throughout this section $c>0$ is fixed.

We henceforth abbreviate $W:=H^{2}(\Omega) \cap H_{0}^{1}(\Omega)$ and define the function $F: W \times L^{2}(\Omega) \times W \rightarrow L^{2}(\Omega)^{3}$ by

$$
F(y, u, p)=\left(\begin{array}{c}
A y+\max _{c}(0, \bar{\lambda}+c(y-\psi))-u \\
A^{*} p+c \operatorname{sgn}_{c}(\bar{\lambda}+c(y-\psi)) p+g^{\prime}(y) \\
j^{\prime}(u)-p
\end{array}\right) .
$$


A generalized derivative [13] $G_{F}$ of $F$ at $(y, u, p) \in W \times L^{2}(\Omega) \times W$ in the direction $(z, h, q) \in W \times L^{2}(\Omega) \times W$ is given by

$$
G_{F}(y, u, p)(z, h, q)=\left(\begin{array}{c}
A z+c \operatorname{sgn}_{c}(\bar{\lambda}+c(y-\psi)) z-h \\
A^{*} q+c \operatorname{sgn}_{c}(\bar{\lambda}+c(y-\psi)) q+c^{2} \operatorname{sgn}_{c}^{\prime}(\bar{\lambda}+c(y-\psi)) p z+g^{\prime \prime}(y) z \\
j^{\prime \prime}(u) h-q
\end{array}\right) .
$$

Theorem 4.1. Let Assumptions 2 and (3.7) be satisfied. Then the semi-smooth Newton method applied to $F(y, u, p)=0$ converges locally superlinearly to $\left(y_{c}, u_{c}, p_{c}\right)$ in $W \times L^{2}(\Omega) \times W$.

The proof uses two preparatory lemmas.

Lemma 4.2. Under the assumptions of Theorem 4.1 the generalized derivatives $G_{F}(y, u, p)$ are uniformly bounded invertible in a $W \times L^{2}(\Omega) \times W$ - neigborhood of $\left(y_{c}, u_{c}, p_{c}\right)$. In particular, the semi-smooth Newton algorithm is well defined in this neighborhood.

Proof. We commence with verifying that the mapping $y \rightarrow \operatorname{sgn}_{c}^{\prime}(\bar{\lambda}+c(y-\psi))$ is continuous at $y=y_{c}$ from $C(\bar{\Omega})$ (and hence from $\left.H^{2}(\Omega)\right)$ to $L^{p}(\Omega)$ for any $p \in[1, \infty)$. In fact, let $w=\bar{\lambda}+c(y-\psi)$ and $w_{c}=\bar{\lambda}+c\left(y_{c}-\psi\right)$. For arbitrary $\delta>0$ let $\left\|y-y_{c}\right\|_{C(\bar{\Omega})} \leq \frac{\delta}{c}$. Then $\left\|w-w_{c}\right\|_{C(\Omega)} \leq \delta$. Note that the set

$$
\mathcal{S}_{\delta}=\left\{x:\left|w_{c}(x)\right| \leq \frac{1}{2 c} \text { and }|w(x)|>\frac{1}{2 c}\right\} \cup\left\{x:\left|w_{c}(x)\right|>\frac{1}{2 c} \text { and }|w(x)| \leq \frac{1}{2 c}\right\}
$$

is contained in

Consequently we find that

$$
\mathcal{T}_{\delta}=\left\{x: \frac{1}{2 c}-\delta \leq\left|w_{c}(x)\right| \leq \frac{1}{2 c}+\delta\right\}
$$

$$
\int_{\Omega}\left|\operatorname{sgn}_{c}^{\prime}(w)-\operatorname{sgn}_{c}^{\prime}\left(w_{c}\right)\right|^{p} \mathrm{~d} x \leq c \operatorname{meas}\left(\mathcal{T}_{\delta}\right) .
$$

By (3.7) we have that meas $\left(\mathcal{T}_{0}\right)=$ meas $\left(\left\{x:\left|\bar{\lambda}+c\left(y_{c}-\psi\right)\right|=\frac{1}{2 c}\right\}\right)=0$. Since the sets $\mathcal{T}_{\delta}$ are monotonically decreasing as $\delta \rightarrow 0^{+}$, we have that $\lim _{\delta \rightarrow 0}$ meas $\left(\mathcal{T}_{\delta}\right)=0$, and hence the desired continuity of $y \rightarrow \operatorname{sgn}_{c}^{\prime}(\bar{\lambda}+$ $c(y-\psi))$ at $y=y_{c}$ follows.

From Assumption 2 and (3.13), it now follows that (3.13) is stable under perturbations in the sense that there exists $\rho>0$ such that

$$
g^{\prime \prime}(y)(z, z)+j^{\prime \prime}(u)(h, h)+\left(c^{2} \operatorname{sgn}_{c}^{\prime}(\bar{\lambda}+c(y-\psi)) p z, z\right) \geq \frac{\alpha}{2}\|h\|_{L^{2}}^{2}
$$

holds for all $(y, u, p)$ with

and all pairs $(h, z)$ satisfying

$$
\left\|y-y_{c}\right\|_{H^{2}}+\left\|u-u_{c}\right\|_{L^{2}}+\left\|p-p_{c}\right\|_{H^{2}} \leq \rho
$$

$$
A z+c \operatorname{sgn}_{c}(\bar{\lambda}+c(y-\psi)) z=h .
$$

Here we use the facts that $x \rightarrow \operatorname{sgn}_{c}(x)$ is globally Lipschitz continuous and that

$$
\left\|z_{y}-z_{y_{c}}\right\|_{H^{2}} \leq K\left\|y-y_{c}\right\|_{L^{\infty}}\|h\|_{L^{2}} \text {, for all } h \in L^{2}(\Omega),
$$

where $z_{y}, z_{y_{c}}$ denote the solutions to (4.4) and (3.9) respectively, and the constant $K$ is uniform with respect to $y$ in bounded subsets of $H^{2}(\Omega)$.

To argue the asserted wellposedness of $G_{F}(y, u, p)$ with $(y, u, p)$ satisfying $(4.3)$ let $r=\left(r_{1}, r_{2}, r_{3}\right) \in L^{2}(\Omega)^{3}$ be arbitrary and consider the equation

for $(z, h, q) \in W \times L^{2}(\Omega) \times W$.

$$
G_{F}(y, u, p)(z, h, q)=r,
$$


For verifying existence of a solution to (4.5) we set

$$
B=A+c \operatorname{sgn}_{c}(\bar{\lambda}+c(y-\psi)) \text { and } b=c^{2} \operatorname{sgn}_{c}^{\prime}(\bar{\lambda}+c(y-\psi)) p
$$

and consider the quadratic minimization problem in $L^{2}(\Omega)$

$$
\begin{gathered}
\min _{h} \frac{1}{2}\left(g^{\prime \prime}(y) B^{-1}\left(h+r_{1}\right), B^{-1}\left(h+r_{1}\right)\right)+\frac{1}{2}\left(b B^{-1}\left(h+r_{1}\right), B^{-1}\left(h+r_{1}\right)\right) \\
+\frac{1}{2}\left(j^{\prime \prime}(u) h, h\right)-\left(r_{2}, B^{-1} h\right)-\left(r_{3}, h\right) .
\end{gathered}
$$

The necessary optimality condition for this problem is

$$
\left(B^{-1}\right)^{*}\left(g^{\prime \prime}(y) B^{-1}\left(h+r_{1}\right)+b B^{-1}\left(h+r_{1}\right)-r_{2}\right)+j^{\prime \prime}(u) h-r_{3}=0,
$$

and the second order sufficient condition is (4.2) with the substitution $B^{-1} h=z$ as in (4.4). Since the second order condition holds for the quadratic problem, (4.6) admits a unique solution. Setting

$$
z=B^{-1}\left(h+r_{1}\right) \text { and } q=-\left(B^{-1}\right)^{*}\left(g^{\prime \prime}(y) z+b z-r_{2}\right)
$$

we have

which is (4.5).

$$
\begin{aligned}
& A z+c \operatorname{sgn}_{c}(\bar{\lambda}+c(y-\psi)) z-h=r_{1}, \\
& A^{*} q+c \operatorname{sgn}_{c}(\bar{\lambda}+c(y-\psi)) q-g^{\prime \prime}(y) z+b z=r_{2}, \\
& j^{\prime \prime}(u) h-q=r_{3},
\end{aligned}
$$

Continuous invertibility of the linear operator

$$
h \rightarrow\left(B^{-1}\right)^{*}\left(g^{\prime \prime}(y) B^{-1} h+b B^{-1} h\right)+j^{\prime \prime}(u) h,
$$

implies the continuous dependence of $h$ on $r$. Continuous dependence of $(z, q)$ on $r$ follows from (4.7).

Lemma 4.3. Let $f: \mathcal{Y} \rightarrow \mathcal{Z}$ and $g: \mathcal{X} \rightarrow \mathcal{Y}$ be Newton differentiable in open sets $V$ and $U$, respectively, with $U \subset \mathcal{X}, g(U) \subset V \subset \mathcal{Y}$. Assume that $g$ is locally Lipschitz continuous and that there exists a Newton map $G_{f}(\cdot)$ of $f$ which is bounded on $g(U)$. Then the superposition $f \circ g: \mathcal{X} \rightarrow \mathcal{Z}$ is Newton differentiable in $U$ with a Newton $\operatorname{map} G_{f} G_{g}$.

For the proof of this lemma we refer to the Appendix of [10].

Proof of Theorem 4.1. According to the superlinear convergence theorem for Newton differentiable mappings $[13,14]$ it suffices to verify that the inverses of the generalized gradients $G_{F}(y, u, p)$ are uniformly bounded in a neighborhood of $\left(y_{c}, u_{c}, p_{c}\right)$, which was achieved in Lemma 4.2 , and that $(y, u, p) \rightarrow F(y, u, p)$ is Newtondifferentiable. This is clear for all terms appearing in $F$ except for $y \rightarrow \operatorname{sgn}_{c}(\bar{\lambda}+c(y-\psi))$ as mapping from $W \rightarrow L^{2}(\Omega)$. Note that

$$
\operatorname{sgn}_{c}(x)=\max \left(1, \max \left(0, c\left(x+\frac{1}{2 c}\right)\right)\right) .
$$

Recall that the max operation is Newton differentiable from $L^{p}(\Omega)$ to $L^{q}(\Omega)$ if $1 \leq q<p$ and

$$
\left(G_{\max } y\right)(x)= \begin{cases}0 & \text { if } y(x)>0 \\ \delta & \text { if } y(x)=0 \\ 1 & \text { if } y(x)<0\end{cases}
$$

for any $\delta \in \mathbb{R}$ and $y \in L^{p}(\Omega)$. Together with Lemma 4.3 this implies Newton differentiability of $y \rightarrow \operatorname{sgn}_{c}(\bar{\lambda}+$ $c(y-\psi))$ from $W \rightarrow L^{2}(\Omega)$. 
Combining the results of Section 3.1 and this section we showed that the solutions of the regularized problem $\left(\mathrm{P}_{c}\right)$ converge in the sense of Proposition 3.8 and that each regularized problem with a fixed value of $c$ can be solved with superlinear rate. In a follow up paper the issue of combining these two asymptotic processes is addressed and the numerical efficiency of this procedure is also established. This will rely on the path $c \rightarrow\left(y_{c}, u_{c}, \lambda_{c}\right)$ associated to $\left(\mathrm{P}_{c}\right)$.

\section{REFERENCES}

[1] V. Barbu, Optimal control of variational inequalities, Monographs and Studies in Mathematics 24. Pitman, Advanced Publishing Program, London (1984).

[2] M. Bergounioux and K. Kunisch, On the structure of Lagrange multipliers for state-constrained optimal control problems. Systems Control Lett. 48 (2003) 169-176.

[3] M. Bergounioux and F. Mignot, Optimal control of obstacle problems: existence of Lagrange multipliers. ESAIM: COCV 5 (2000) $45-70$.

[4] H. Brezis and G. Stampacchia, Sur la régularité de la solution d'inéquations elliptiques. Bull. Soc. Math. France 96 (1968) 153-180.

[5] E. Casas and M. Mateos, Second order optimality conditions for semilinear elliptic control problems with finitely many state constraints. SIAM J. Control Optim. 40 (2002) 1431-1454.

[6] E. Casas, F. Tröltzsch and A. Unger, Second order sufficient optimality conditions for some state-constrained control problems of semilinear elliptic equations. SIAM J. Control Optim. 38 (2000) 1369-1391.

[7] E. Casas, J.C. de los Reyes and F. Tröltzsch, Sufficient second-order optimality conditions for semilinear control problems with pointwise state constraints. SIAM J. Optim. 19 (2008) 616-643.

[8] P. Grisvard, Elliptic problems in nonsmooth domains, Monographs and Studies in Mathematics 24. Pitman, Advanced Publishing Program, Boston, MA (1985).

[9] M. Hintermüller and I. Kopacka, Mathematical programs with complementarity constraints in function space: $C$ - and strong stationarity and a path-following algorithm. SIAM J. Optim. 20 (2009) 868-902.

[10] M. Hintermüller and K. Kunisch, Pde-constrained optimization subject to pointwise control and zero- or first-order state constraints. SIAM J. Optim. 17 (2006) 159-187.

[11] K. Ito and K. Kunisch, An augmented Lagrangian technique for variational inequalities. Appl. Math. Optim. 21 (1990) $223-241$.

[12] K. Ito and K. Kunisch, Optimal control of elliptic variational inequalities. Appl. Math. Optim. 41 (2000) 343-364.

[13] K. Ito and K. Kunisch, Semi-smooth Newton methods for variational inequalities of the first kind. ESAIM: M2AN 37 (2003) $41-62$.

[14] K. Ito and K. Kunisch, On the Lagrange multiplier approach to variational problems and applications, Monographs and Studies in Mathematics 24. SIAM, Philadelphia (2008).

[15] F. Mignot, Contrôle dans les inéquations variationelles elliptiques. J. Funct. Anal. 22 (1976) 130-185.

[16] F. Mignot and J.-P. Puel, Optimal control in some variational inequalities. SIAM J. Control Optim. 22 (1984) $466-476$.

[17] J.-P. Raymond and F. Tröltzsch, Second order sufficient optimality conditions for nonlinear parabolic control problems with state constraints. Discrete Contin. Dyn. Syst. 6 (2000) 431-450.

[18] A. Rösch and F. Tröltzsch, Sufficient second-order optimality conditions for an elliptic optimal control problem with pointwise control-state constraints. SIAM J. Optim. 17 (2006) 776-794.

[19] H. Scheel and S. Scholtes, Mathematical programs with complementarity constraints: stationarity, optimality, and sensitivity. Math. Oper. Res. 25 (2000) 1-22.

[20] G. Stampacchia, Le problème de Dirichlet pour les équations elliptiques du second ordre à coefficients discontinus. Ann. Inst. Fourier (Grenoble) 15 (1965) 189-258.

[21] F. Tröltzsch, Optimale Steurung partieller Differentialgleichungen. Vieweg + Teubner, Wiesbaden (2009).

[22] K. Yosida and E. Hewitt, Finitely additive measures. Trans. Am. Math. Soc. 72 (1952) 46-66. 